\title{
High-brilliance, high-flux compact inverse Compton light source
}

\author{
K. E. Deitrick, ${ }^{1, *}$ G. A. Krafft, ${ }^{1,2}$ B. Terzić, ${ }^{1}$ and J. R. Delayen ${ }^{1,2}$ \\ ${ }^{1}$ Department of Physics, Center for Accelerator Science, Old Dominion University, \\ Norfolk, Virginia 23529, USA \\ ${ }^{2}$ Thomas Jefferson National Accelerator Facility, Newport News, Virginia 23606, USA
}

(Received 29 March 2018; published 24 August 2018)

\begin{abstract}
The Old Dominion University Compact Light Source (ODU CLS) design concept is presented-a compact Inverse Compton Light Source (ICLS) with flux and brilliance orders of magnitude beyond conventional laboratory-scale sources and greater than other compact ICLS designs. This concept utilizes the physics of inverse Compton scattering of an extremely low emittance electron beam by a laser pulse of $r m s$ length of approximately two-thirds of a picosecond $(2 / 3 \mathrm{ps})$. The accelerator is composed of a superconducting radio frequency (SRF) reentrant gun followed by four double-spoke SRF cavities. After the linac are three quadrupole magnets to focus the electron beam to the interaction point (IP). The distance from cathode surface to IP is less than $6 \mathrm{~m}$, with the cathode producing electron bunches with a bunch charge of $10 \mathrm{pC}$ and a few picoseconds in length. The incident laser has $1 \mathrm{MW}$ circulating power, a 1 micron wavelength, and a spot size of 3.2 microns at the IP. The repetition rate of this source is $100 \mathrm{MHz}$, in order to achieve a high flux despite the low bunch charge. The anticipated $\mathrm{x}$-ray source parameters include an energy of $12 \mathrm{keV}$, with a total flux of $2.2 \times 10^{13} \mathrm{ph} / \mathrm{s}$, the flux into a $0.1 \%$ bandwidth of $3.3 \times 10^{10} \mathrm{ph} /(\mathrm{s} 0.1 \% \mathrm{BW})$, and the average brilliance of $3.4 \times 10^{14} \mathrm{ph} /\left(\mathrm{s} \mathrm{mm}^{2} \mathrm{mrad}^{2} 0.1 \% \mathrm{BW}\right)$.
\end{abstract}

DOI: 10.1103/PhysRevAccelBeams.21.080703

\section{INTRODUCTION}

Since their discovery in 1895 , $\mathrm{x}$-rays have been a powerful technique for determining the structure of condensed matter. For the first 70 years of using x-rays, sources barely changed from the original bremsstrahlung tubes used in their discovery $[1,2]$. Until recently, large accelerator-based synchrotron facilities set the standard for the highest quality $\mathrm{x}$-ray beams. At present, this standard has been largely surpassed in free electron lasers (FELs).

Most high-brilliance sources exist at large facilities, especially third-generation synchrotrons [3]. However, due to various concerns, among them cost, risk of transporting valuable items, and limited available runtime at large facilities, there has been an increasing demand for laboratory-scale sources. Additional desirable constraints are that the purchase and operating cost are not prohibitive for the smaller facilities and that the operation of a such a machine is possible by nonexperts.

There are many $\mathrm{x}$-ray experimental techniques that exist today; any given technique may be utilized in a wide range

\footnotetext{
*kdeit001@odu.edu, deitrick@jlab.org Present address: Jefferson Lab, Virginia, USA.

Published by the American Physical Society under the terms of the Creative Commons Attribution 4.0 International license. Further distribution of this work must maintain attribution to the author(s) and the published article's title, journal citation, and DOI.
}

of fields. Some of the more prominent techniques currently in use include phase contrast imaging (PCI), absorption radiography, K-edge subtraction imaging, radiotherapy (treatment of tumors with $\mathrm{x}$-rays), and computed tomography (CT). Some of the fields in which these techniques are used include medicine, cultural heritage, material science development, and industry $[4,5]$. For this concept, the primary applications are in the area of cultural heritage and materials science. It would allow investigation of materials at atomic scale, and the dynamics of systems at femtosecond scale. It is also of a size suitable for universities and small institutions, thus contributing to the education of the next generation of scientists and bringing this powerful scientific tool and technique to a wider community [2]. Given the wide range of applications, the increasing demand for higher quality $\mathrm{x}$-ray sources is understandable. In this paper, we present a design of a compact Inverse Compton Source based on SRF beam acceleration which was outlined in [6]. Because the SRF is run continuous wave $(\mathrm{CW})$, high average flux and brilliance are possible. While we have chosen $12 \mathrm{keV}$ as the top $\mathrm{x}$-ray energy for this design, the top energy (and the number of potential applications) can be increased by extending the linac. For example, the addition of a cryomodule of two cavities would raise the top x-ray energy to $30 \mathrm{keV}$. This addition would also increase the average flux and brilliance of the x-rays generated.

This paper is organized as follows. A brief overview of Inverse Compton Light Sources, inverse Compton scattering, 


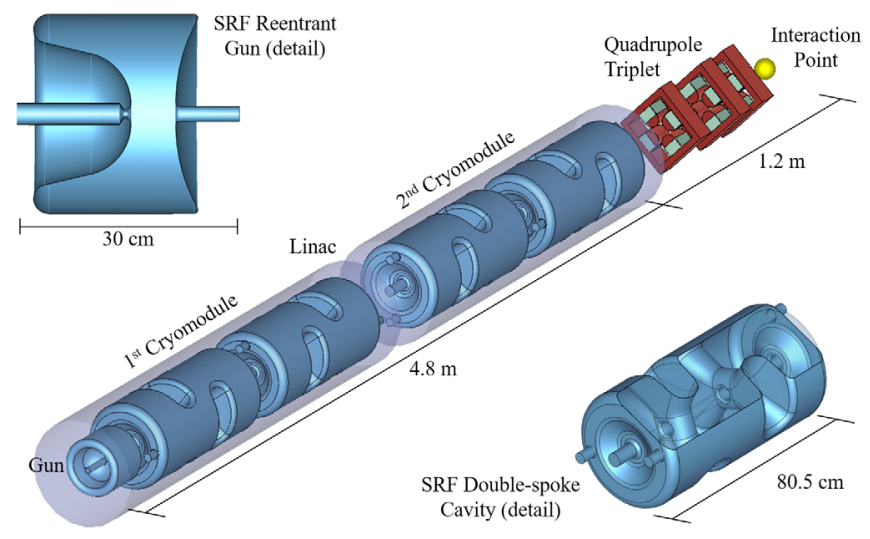

FIG. 1. A schematic of the entire design. The first cryomodule contains the gun and two double-spoke cavities, the second contains the last two double-spoke cavities. Three quadrupole magnets follow the linac, before the interaction point. Detailed images of the gun and the double-spoke cavity are shown in the top left and bottom right, respectively.

and compact ICLS designs is presented in Sec. II. Our design concept consists of three separate regions: the SRF reentrant gun (Sec. III), the SRF linear accelerator (Sec. IV), and the final focusing (Sec. V). A complete layout of these design components is shown in Fig. 1. In Sec. VI the sensitivity study results are presented, while the incident laser is addressed in
Sec. VII. The complete $\mathrm{x}$-ray beam parameters are presented in Sec. VIII, plans for future work are given in Sec. IX, before the final summary presented in Sec. X. The design presented in this paper potentially outperforms all other compact ICLS designs.

\section{INVERSE COMPTON LIGHT SOURCE}

\section{A. Inverse Compton scattering}

The process of scattering a photon off an electron at rest is known as Compton scattering. The term inverse Compton scattering (ICS) is used in the situation such that the electron loses energy to the incident photons. In the following formulas, $\Phi$ is the angle between the relativistic electron and the laser beams, and $\Delta \Theta$ is the angle between the laser beam and scattered photons. If $\theta$ and $\phi$ represent spherical polar angles that the scattered photons make in the coordinate system such that the electron beam moves along the $z$ axis, then the angle $\Delta \Theta$ is $\cos \Delta \Theta=$ $\cos \Phi \cos \theta-\sin \Phi \sin \theta \cos \phi$. The coordinate system is set so the interaction point (IP) of the electron and laser beams occurs in the $x-z$ plane.

A general formula expressing the energy of a scattered photon in the lab frame, $E_{\gamma}$, as a function of the direction of the scattered photon, is

$$
E_{\gamma}(\Phi, \theta, \phi)=\frac{E_{\text {laser }}(1-\beta \cos \Phi)}{1-\beta \cos \theta+E_{\text {laser }}(1-\cos \Phi \cos \theta+\sin \Phi \sin \theta \cos \phi) / E_{e^{-}}}
$$

where $\beta$ is the relativistic factor $v_{z} / c, E_{\text {laser }}$ is the energy of the typical laser photon, and $E_{e^{-}}=\gamma m_{e} c^{2}$ is the energy of the electron, with $\gamma$ the usual relativistic factor [5]. This formula includes the impact of electron recoil. The Thomson formula is a good approximation if the electron recoil is negligible, i.e., the energy of the laser in the beam frame is much less than the rest mass of the electron. When this is true, then the formula for the energy of the scattered photon becomes

$$
E_{\gamma}(\Phi, \theta) \approx E_{\text {laser }} \frac{1-\beta \cos \Phi}{1-\beta \cos \theta} .
$$

Consider the situation of a head-on collision between the electron and the laser $(\Phi=\pi)$. Assuming that the Thomson formula is a good approximation, i.e., $E_{\text {laser }}^{\prime}=$ $\gamma(1+\beta) E_{\text {laser }} \ll m c^{2}$ is true, then the energy of the scattered photon is also $E_{\text {laser }}^{\prime}$ in the beam frame. Going back into the lab frame, the photons scattered in the forward (positive $z$ ) direction have the highest energy, which is $\gamma^{2}(1+\beta)^{2} E_{\text {laser }} \approx 4 \gamma^{2} E_{\text {laser. }}$. The high energy boundary of emission is called the Compton edge; no radiation is emitted at higher energies. For photons scattered at the angle $\theta$ such that $\sin \theta=1 / \gamma \ll 1$, the energy decreases to
$2 \gamma^{2} E_{\text {laser }}$, which is also the average energy of the scattered photons. Both the Compton edge and the number density of scattered photons as a function of the energy of scattered photons can be seen in Fig. 2.

The number of photons produced by scattering an incident laser off an electron is proportional to the

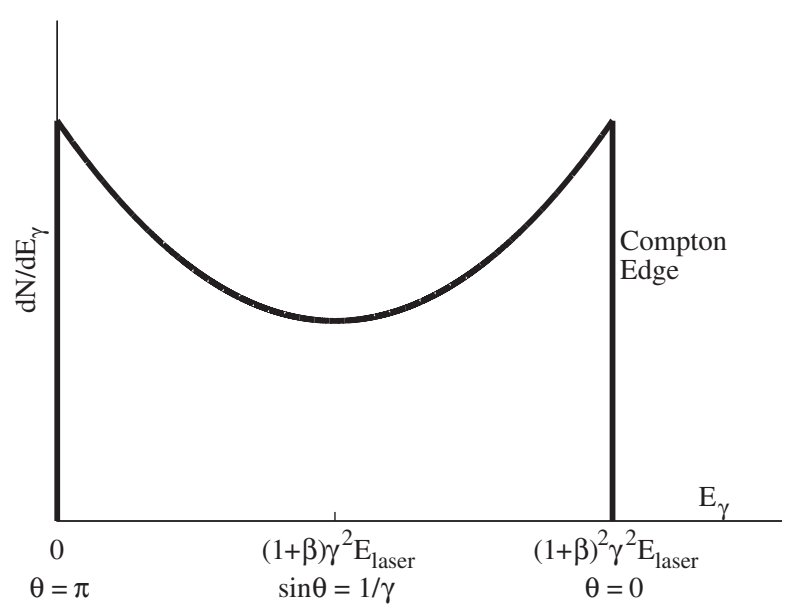

FIG. 2. Number density of scattered photons as a function of the energy of scattered photons. Annotated Fig. 2 from [5]. 
time-integrated intensity of illumination. Consequently, the total photon yield is proportional to the square of the field strength, as in the case of undulator radiation. Progressing by the analogy with undulator radiation, the field strength parameter for a plane wave incident laser is defined to be

$$
a=\frac{e E \lambda_{\text {laser }}}{2 \pi m c^{2}},
$$

where $e$ is the electron charge, $E$ is the transverse electric field of the laser, $\lambda_{\text {laser }}$ is the laser wavelength, and $m c^{2}$ is the rest energy of the electron. This value represents the normalized transverse vector potential for the electromagnetic (EM) field accelerating the electrons during scattering. For Compton scattering, $a$ plays a role similar to that of $K$ in the field of undulators. For the case of $a \ll 1$, the backscattering is in the linear regime, an assumption that continues as formulae are presented.

If we take the assumption that the transverse intensity distributions of the laser and electron beams are round Gaussian distributions with the rms sizes of $\sigma_{e}$ and $\sigma_{\text {laser }}$ respectively, then

$$
U_{\gamma}=\gamma^{2}(1+\beta) \sigma_{T} \frac{N_{e} N_{\text {laser }}}{2 \pi\left(\sigma_{e}^{2}+\sigma_{\text {laser }}^{2}\right)} E_{\text {laser }},
$$

where $U_{\gamma}$ is the total energy of the scattered photons per collision, $N_{e}$ is the number of electrons in the bunch, $N_{\text {laser }}$ the number of photons in the incident laser, and $\sigma_{T}$ is the Thomson cross section of $6.65 \times 10^{-29} \mathrm{~m}^{2}$. From this formula, the total number of scattered photons $N_{\gamma}$ is

$$
N_{\gamma}=\sigma_{T} \frac{N_{e} N_{\text {laser }}}{2 \pi\left(\sigma_{e}^{2}+\sigma_{\text {laser }}^{2}\right)} .
$$

Given that the spectral energy density of the scattered photons may be analytically computed in the linear Thomson backscatter limit, it can further be determined that the number of scattered photons within a $0.1 \%$ bandwidth at the Compton edge is $N_{0.1 \%}=1.5 \times 10^{-3} N_{\gamma}$. Consequently, the rate of photons (flux) into this bandwidth is $\mathcal{F}_{0.1 \%}=$ $1.5 \times 10^{-3} \dot{N}_{\gamma}$. For high-frequency repetitive sources, $\dot{N}_{\gamma}=f N_{\gamma}$, where $f$ is the repetition rate $[5,7,8]$.

The spectral brightness or brilliance of a photon beam is the density of photons in the six-dimensional space containing the beam. The general formula for the brilliance of a photon beam into a $0.1 \%$ bandwidth is

$$
\mathcal{B}=\frac{\mathcal{F}_{0.1 \%}}{4 \pi^{2} \sigma_{\gamma, x} \sigma_{\gamma, x^{\prime}} \sigma_{\gamma, y} \sigma_{\gamma, y^{\prime}}}
$$

where $\sigma_{\gamma, x}$ and $\sigma_{\gamma, y}$ are the rms transverse sizes of the photon beam and $\sigma_{\gamma, x^{\prime}}$ and $\sigma_{\gamma, y^{\prime}}$ are the rms transverse angular sizes of the photon beam. However, by taking advantage of the analogy to undulator radiation, it is possible to approximate the brilliance of the scattered photons using the parameters of the electron beam at the collision. The standard approximation for a nondiffraction limited beam is $\sigma_{\gamma, x^{\prime}} \approx \sqrt{\epsilon_{x} / \beta_{x}}$, where $\epsilon_{x}$ and $\beta_{x}$ are parameters of the electron beam [5]. Taking this approximation into account, Eq. (6) becomes

$$
\mathcal{B}=\frac{\mathcal{F}_{0.1 \%}}{4 \pi^{2} \sigma_{\gamma, x} \sqrt{\epsilon_{x} / \beta_{x}} \sigma_{\gamma, y} \sqrt{\epsilon_{y} / \beta_{y}}} .
$$

In previous papers [9-11], we have taken the approximation that the $\mathrm{x}$-ray source size is the size of the electron beam; this approximation is typical in the characterization of compact sources $[4,5]$. In this approach, $\sigma_{\gamma, x}=\sigma_{x}=\sqrt{\beta_{x} \epsilon_{x}}$, so Eq. (7) becomes

$$
\mathcal{B} \approx \frac{\gamma^{2} \mathcal{F}_{0.1 \%}}{4 \pi^{2} \epsilon_{x, \mathrm{rms}}^{N} \epsilon_{y, \mathrm{rms}}^{N}}
$$

If instead we take the position that the source size is a convolution of the electron and laser beam sizes, such that

$$
\frac{1}{\sigma_{\gamma}^{2}}=\frac{1}{\sigma_{\text {laser }}^{2}}+\frac{1}{\sigma_{x} \sigma_{y}} .
$$

Using this, Eq. (7) becomes

$$
\mathcal{B} \approx \frac{\gamma \mathcal{F}_{0.1 \%}}{4 \pi^{2} \sigma_{\gamma}^{2} \sqrt{\epsilon_{x, \text { rms }}^{N} \epsilon_{y, \text { rms }}^{N} / \beta_{x} \beta_{y}}} .
$$

As the laser spot size becomes increasingly greater than the electron beam spot size, the difference between Eqs. (8) and (10) becomes negligible. However, for the compact source presented in this paper, the spot sizes are roughly equivalent, making Eq. (10) more appropriate. From either brilliance formula, it becomes clear that to maximize brilliance requires maximizing the photon flux, maximizing the electron beam energy, or minimizing the electron beam normalized rms transverse emittances.

The bandwidth of the scattered radiation from a head-on collision in the forward direction through a small aperture for the regime where recoil is negligible is given by

$$
\frac{\sigma_{E_{\gamma}}}{E_{\gamma}}=\sqrt{\left(\frac{\sigma_{E_{\theta_{\max }}}}{E_{\theta_{\max }}}\right)^{2}+\left(2 \frac{\sigma_{E_{e}}}{E_{e}}\right)^{2}+\left(\frac{\sigma_{E_{l}}}{E_{l}}\right)^{2}+\left(\frac{\sigma_{E_{e}}}{E_{\epsilon}}\right)^{2}}
$$

where $\sigma_{E_{e}} / E_{e}$ and $\sigma_{E_{l}} / E_{l}$ are the relative energy spreads of the electron beam and the laser beam, respectively. The contribution due to the small aperture is given by

$$
\frac{\sigma_{E_{\theta_{\max }}}}{E_{\theta_{\max }}}=\frac{1}{\sqrt{12}} \frac{\gamma^{2} \theta_{\max }^{2}}{1+\gamma^{2} \theta_{\max }^{2} / 2},
$$

while the contribution due to the electron beam emittance through a small aperture is given by 


$$
\frac{\sigma_{E_{\epsilon}}}{E_{\epsilon}}=\sqrt{2} \gamma \sqrt{\frac{\epsilon_{x}^{2}}{\beta_{x}^{2}}+\frac{\epsilon_{y}^{2}}{\beta_{y}^{2}}},
$$

where all parameters are of the electron beam at the interaction point. This is an extremely specific formulation-the more generalized formulation can be found in [12].

\section{B. Compact Sources}

There are two main components in an inverse Compton light source (ICLS) - a relativistic electron beam and an incident laser. In the last several years, there has been a significant advancement in the technology to produce a suitable laser. The details of this progress are largely beyond the scope of this article, though the status of the current technology will be touched on later. The other component, the focus of this concept, is the relativistic electron beam off which the incident laser scatters.

There exist two schemes for accelerating an electron beam to the desired energy, typically in the range of a few $10 \mathrm{~s}$ of MeV: a linear accelerator (linac) or a storage ring (ring) [4]. A linac is composed of radiofrequency (rf) or superconducting (SC) rf (SRF) cavities that accelerate the beam to the desired energy [13]. Rings are circular devices into which a beam of a specific energy is injected, where the beam may or may not be extracted before being used [14].

Both of these options have benefits and drawbacks. Existing storage ring projects typically have lower expected fluxes than those of linacs. The expected brightness is frequently lower [4], as the smallest achievable normalized emittances are typically larger for a ring than a linac. Additionally, a full energy linac is often required anyway for injection into the ring $[2,4,13]$. However, rings are capable of a high repetition rate, a higher average current than is typical for linacs, and historically have better stability $[2,4]$.

Linac-based ICS x-ray sources have shown promising results at lower pulse repetition rates, though these results have yet to be reproduced at higher rates. For electron beams with an energy above $10 \mathrm{MeV}$, cumbersome shielding must be included [2,4]. Current cryogenic equipment for SRF structures, which are by some assumed to be necessary for a linac project to succeed, are more complicated than nonexpert users are comfortable using. Another common feature to most linac projects is a superconducting electron gun, a technology with promising results but not yet a mature field $[4,15]$. Linac projects are more likely to be capable of shorter bunch lengths, even without compression, smaller normalized emittances, and a greater flexibility for phase space manipulations than ring projects $[2,4]$. Shorter bunch lengths are important to avoid degradation of the electron beam during bunch compression and because longer electron bunches at the IP result in a reduction of the X-ray beam due to the hourglass effect.
Referenced in the literature as the first existing compact Compton source is the one built by Lyncean Technologies. An electron beam is produced by a normal conducting linac and injected into a storage ring, which occupies a $1 \mathrm{~m}$ by $2 \mathrm{~m}$ footprint. This machine delivers $\sim 10^{9} \mathrm{ph} / \mathrm{s}$ in a $3 \%$ energy bandwidth, with the scattered photon beam having an $r m s$ spot size of $\sim 45 \mu \mathrm{m}[4,16]$. This became the first commercially sold ICS light source, recently installed at the Technische Universität München in Munich, Germanythe Munich Compact Light Source (MuCLS). It has a demonstrated performance of a $\sim 42 \mu \mathrm{m}$ spot size, a total average flux of $10^{9}-10^{10} \mathrm{ph} / \mathrm{s}$, and an average brilliance of $4.8 \times 10^{9}$ photons $/\left(\mathrm{s} \mathrm{mm}^{2} \mathrm{mrad}^{2} 0.1 \% \mathrm{BW}\right)$ [17].

Current $\mathrm{x}$-ray flux expectations of compact sources are on the order of $10^{12}-10^{13} \mathrm{ph} / \mathrm{s}$ for storage ring projects and $10^{13}-10^{14} \mathrm{ph} / \mathrm{s}$ for linac projects, with an anticipated brilliance on the order of $10^{12}-10^{15} \mathrm{ph} /$ $\left(\mathrm{s} \mathrm{mm}^{2} \mathrm{mrad}^{2} 0.1 \% \mathrm{BW}\right)$ for linac projects [4]. To give some perspective to these values, the best rotating anodes, such as may currently be found in a lab as an $\mathrm{x}$-ray source, have a flux of $\sim 6 \times 10^{9} \mathrm{ph} / \mathrm{s}$ and a brightness on the order of $10^{9}$ photons $/\left(\mathrm{s} \mathrm{mm}^{2} \mathrm{mrad}^{2} 0.1 \% \mathrm{BW}\right)$ [2]. On the other hand, an x-ray beam that might typically be found at a large facility has a flux in the regime of $\sim 10^{11}-10^{13} \mathrm{ph} / \mathrm{s}$ [18] and a brightness of $\sim 10^{19}$ photons/ ( $\mathrm{smm}^{2} \mathrm{mrad}^{2} 0.1 \% \mathrm{BW}$ ) [19].

Given these numbers, a robust user program for a compact ICLS machine would require that substantial fluxes of narrow-band x-rays are the desired requirement, rather than the best average brightness. However, the potential for such machines, in terms of both performance and demand, make the prospect of a well-designed compact source non-negligible [5].

\section{Considerations for this concept and design parameters choices}

The main goal of this study was to develop the concept for a high-brilliance, high-flux inverse Compton light source that would also be relatively affordable and easy to operate by nonexperts. High-flux would imply cw operation and an SRF linac, and ease of operation would suggest operating with atmospheric helium at $4.2 \mathrm{~K}$ or above. Since the surface resistance of superconductors increases quadratically with frequency this would imply a low-frequency system. On the other hand, the size and cost of the cavities and cryomodules increase as the frequency is lowered, and a trade-off between the two considerations suggested a frequency range of 300 to $500 \mathrm{MHz}$ [9].

A number of accelerating structure geometries were considered. The most common and widespread is the transverse magnetic (TM) cavity, sometimes referred to as "elliptical." This geometry is well understood and has the advantage of having rotational symmetry. However it was deemed to be too large in that frequency range. Another 
type of superconducting structure is the spoke geometry which, at the same frequency, is smaller than the TM-type $[20,21]$. Several of these cavities have been developed in the frequency and velocity range of interest [22]. A $325 \mathrm{MHz}$ single-spoke cavity had been successfully developed but was also deemed to be too large [23]. We finally decided on a $500 \mathrm{MHz}$, double-spoke geometry which had also been successfully developed [24]. The spoke geometry has the disadvantage of introducing quadrupole components in the electromagnetic fields [25]. As shown later, the contribution of the quadrupole components can be managed and its impact on the final performance is minimal.

We would like to emphasize that further advances in the SRF technology could justify revisiting the geometry and/or frequency choice but would not invalidate the conclusions of this study.

To attain a high average flux, considering that the average flux is proportional to both the bunch charge and the repetition rate, a high repetition rate of $100 \mathrm{MHz}$ was chosen to counterbalance the low bunch charge. Minimizing the spot size of both electron and laser beams also helped to increase the flux. Thus, the spot size for the electron beam at the IP was set at $\sim 3 \mu \mathrm{m}$, which is small but feasible, though it will require state-of-the-art diagnostics at the IP. To increase brightness for a given flux and electron spot size, the normalized rms transverse emittance needs to be minimized, leading to a target value of $0.1 \mathrm{~mm}$ mrad. While this value is considerably smaller than in other SRF injector guns, as shown in this work, a low bunch charge of $10 \mathrm{pC}$ makes this emittance attainable $[9,15]$.

An electron beam energy of $25 \mathrm{MeV}$ and an incident scattering laser energy of $1.24 \mathrm{eV}$ were chosen. The chosen energies generate $\mathrm{x}$-rays of up to $12 \mathrm{keV}$. X-rays at $12 \mathrm{keV}$ have a corresponding wavelength of approximately one Angström, the same as in large third generation synchrotron facilities. For the energy smearing of the forward flux to be small relative to the total bandwidth necessitates that the relative beam energy spread be less than $0.03 \%$. At the chosen energy of $25 \mathrm{MeV}$, this leads to an rms energy spread requirement of $7.5 \mathrm{keV}$. In order to keep the flux reduction due to the hourglass effect negligible, the compressed bunch length is set to less than $1 \mathrm{~mm}$ [9].

For the best possible $\mathrm{x}$-ray beam, a high quality high power laser is necessary. The ideal laser would, among other properties, have a circulating power of $1 \mathrm{MW}$, compared to $100 \mathrm{~kW}$ today. One MW is widely regarded as feasible, but has not yet been achieved in a compact optical cavity $[4,5,9,26]$. The other properties relevant to the optical cavity are less demanding: $1 \mu \mathrm{m}$ wavelength $(1.24 \mathrm{eV}), 5 \times 10^{16} \mathrm{ph} /$ bunch, spot size of $3.2 \mu \mathrm{m}$ at collision, and peak strength parameter $a=0.026$ [9]. However, a $3.2 \mu \mathrm{m}$ laser spot size has an extremely short Rayleigh range (which presents additional challenges), so results are also presented for a laser spot size of $12 \mu \mathrm{m}$, while keeping the electron spot size at $3.2 \mu \mathrm{m}$.
TABLE I. Desired electron beam parameters at interaction point.

\begin{tabular}{lcc}
\hline \hline Parameter & Quantity & Units \\
\hline Kinetic energy & 25 & $\mathrm{MeV}$ \\
Bunch charge & 10 & $\mathrm{pC}$ \\
Repetition rate & 100 & $\mathrm{MHz}$ \\
Average current & 1 & $\mathrm{~mA}$ \\
Transverse normalized $r m s$ emittance & 0.1 & $\mathrm{~mm} \mathrm{mrad}$ \\
$\beta_{x, y}$ & 5 & $\mathrm{~mm}$ \\
$\sigma_{x, y}$ & 3 & $\mu \mathrm{m}$ \\
FWHM bunch length & $3(0.9)$ & $\mathrm{ps}(\mathrm{mm})$ \\
$r m s$ energy spread & 7.5 & $\mathrm{keV}$ \\
\hline \hline
\end{tabular}

These specifications are based on and similar to those earlier presented in [26]. A summary of the desired electron beam parameters at the interaction point (IP) are shown in Table I. A summary of optical cavity parameters are shown in Table II, based on performances that may soon be attainable $[5,26]$.

It is possible to take the properties of the electron beam and incident laser beam and estimate selected parameters of the x-ray beam which would be produced from a collision between the two, using formulae presented previously. For a laser spot size of $3.2 \mu \mathrm{m}$ and electron spot size of $3.2 \mu \mathrm{m}$, the $\mathrm{x}$-ray beam energy will be $12 \mathrm{keV}$ with $1.6 \times 10^{6}$ photons/bunch. The $\mathrm{x}$-ray beam flux will be $1.6 \times 10^{14} \mathrm{ph} / \mathrm{s}$, with an average brilliance of $3 \times 10^{15} \mathrm{ph} /\left(\mathrm{s} \mathrm{mm}^{2} \mathrm{mrad}^{2} 0.1 \% \mathrm{BW}\right)$. For a laser spot size of $12 \mu \mathrm{m}$ and electron spot size of $3.2 \mu \mathrm{m}$, the $\mathrm{x}$-ray beam energy will be $12 \mathrm{keV}$ with $2.1 \times 10^{5}$ photons/bunch. The $\mathrm{x}$-ray beam flux will be $2.1 \times 10^{13} \mathrm{ph} / \mathrm{s}$, with an average brilliance of $2.1 \times 10^{14} \mathrm{ph} /\left(\mathrm{s} \mathrm{mm}^{2} \mathrm{mrad}^{2} 0.1 \% \mathrm{BW}\right)$.

However, the extremely short Rayleigh range due to the laser spot size means that diffraction effects need to be taken into account. Using the formulae given in [27], we have calculated the anticipated reduction for the resulting x-rays associated with both laser spot sizes. The corresponding reduction is $85.25 \%$ and $25.73 \%$, for the 3.2 and $12 \mu \mathrm{m}$ laser spot sizes, respectively. Taking this reduction into account, for a laser spot size of $3.2 \mu \mathrm{m}$ and electron spot size of $3.2 \mu \mathrm{m}$, the x-ray beam energy will be $12 \mathrm{keV}$ with $2.4 \times 10^{5}$ photons/bunch. The $\mathrm{x}$-ray beam flux will be $2.4 \times 10^{13} \mathrm{ph} / \mathrm{s}$, with an average brilliance of

TABLE II. Laser parameters at interaction point.

\begin{tabular}{lcc}
\hline \hline Parameter & Quantity & Units \\
\hline Wavelength & $1(1.24)$ & $\mu \mathrm{m}(\mathrm{eV})$ \\
Circulating power & 1 & $\mathrm{MW}$ \\
$N_{\gamma}$, Number of photons/bunch & $5 \times 10^{16}$ & \\
Spot size $(r m s)$ & $3.2,12$ & $\mu \mathrm{m}$ \\
Peak strength parameter, $a$ & $0.026,0.002$ & \\
$\quad a=e E \lambda_{\text {laser }} / 2 \pi m c^{2}$ & 100 & $\mathrm{MHz}$ \\
Repetition rate & $2 / 3$ & $\mathrm{ps}$ \\
$r m s$ pulse duration &
\end{tabular}


TABLE III. Desired light source parameters. Flux and brilliance reported for top x-ray energy, reduced for lower energies.

\begin{tabular}{lccc}
\hline \hline & \multicolumn{2}{c}{ Laser spot $(\mu \mathrm{m})$} & \\
\cline { 2 - 4 } Parameter & 3.2 & 12 & Units \\
\hline X-ray energy & $1.2-12$ & $1.2-12$ & $\mathrm{keV}$ \\
Photons/bunch & $2.4 \times 10^{5}$ & $1.6 \times 10^{5}$ & $\mathrm{ph} / \mathrm{s}$ \\
Flux & $2.4 \times 10^{13}$ & $1.6 \times 10^{13}$ & $\mathrm{ph} /\left(\mathrm{s} \mathrm{mm}^{2} \mathrm{mrad}^{2} 0.1 \% \mathrm{BW}\right)$ \\
Average brilliance & $4.4 \times 10^{14}$ & $1.6 \times 10^{14}$ & \\
\hline \hline
\end{tabular}

TABLE IV. Comparison of x-ray beam parameters for different ICLS compact designs. ${ }^{e}$ indicates existing designs, $f$ indicates funded designs; (SC) indicates superconducting cavities. Versions of this table and some information within were published previously by Jacquet [6] and Jacquet and Suortti [28]. The bolded lines are the anticipated x-ray beam parameters for the concept presented in this paper using a scattering laser with a 3.2 micron $\left(\sigma_{\text {laser }}=3.2 \mu \mathrm{m}\right)$ and 12 micron $\left(\sigma_{\text {laser }}=12 \mu \mathrm{m}\right)$ spot size.

\begin{tabular}{|c|c|c|c|c|c|c|}
\hline Design & Type & $E_{x}(\mathrm{keV})$ & $\mathrm{Ph} / \mathrm{s}$ & $\mathrm{Ph} /\left(\mathrm{s} \mathrm{mrad}^{2} \mathrm{~mm}^{2} 0.1 \% \mathrm{BW}\right)$ & Repetition Rate $(\mathrm{MHz})$ & $\sigma_{\gamma}(\mu \mathrm{m})$ \\
\hline${ }^{e} \mathrm{MuCLS}$ [17] & SR & $15-35$ & $10^{10}$ & $10^{9}$ & 65 & 42 \\
\hline${ }^{e}$ Lyncean $[5,16]$ & SR & $10-20$ & $10^{11}$ & $10^{11}$ & 65 & 45 \\
\hline TTX [29] & SR & $20-80$ & $10^{12}$ & $10^{10}$ & 75 & 50 \\
\hline${ }^{f}$ BriXS, collimated [30] & Linac (SC) & $83-88$ & $10^{12}$ & $10^{12}$ & 100 & 14 \\
\hline LEXG [31] & $\mathrm{SR}(\mathrm{SC})$ & 33 & $10^{13}$ & $10^{11}$ & 0.001 & 20 \\
\hline${ }^{e}$ ThomX $[32,33]$ & SR & $20-90$ & $10^{13}$ & $10^{11}$ & 33.4 & 70 \\
\hline${ }^{e} \mathrm{KEK}$ QB [34] & Linac (SC) & 35 & $10^{13}$ & $10^{11}$ & 0.005 & 10 \\
\hline${ }^{e} \mathrm{KEK}$ ERL [35] & Linac (SC) & 67 & $10^{13}$ & $10^{11}$ & 130 & 30 \\
\hline${ }^{e}$ NESTOR [36] & SR & $30-500$ & $10^{13}$ & $10^{12}$ & 0.01 & 70 \\
\hline${ }^{f}$ ASU (MIT) [2] & Linac & 12 & $10^{13}$ & $10^{12}$ & 0.001 & 2 \\
\hline${ }^{f}$ BriXS, uncollimated [30] & Linac (SC) & $83-88$ & $10^{13}$ & $10^{13}$ & 100 & 14 \\
\hline ODU CLS $\left(\sigma_{\text {laser }}=12 \mu \mathrm{m}\right)$ & Linac (SC) & $1.2-12$ & $10^{13}$ & $10^{14}$ & 100 & 3 \\
\hline ODU CLS $\left(\sigma_{\text {laser }}=3.2 \mu \mathrm{m}\right)$ & Linac (SC) & $1.2-12$ & $10^{13}$ & $10^{14}$ & 100 & 2 \\
\hline
\end{tabular}

$4.4 \times 10^{14} \mathrm{ph} /\left(\mathrm{s} \mathrm{mm}^{2} \mathrm{mrad}^{2} 0.1 \% \mathrm{BW}\right)$. For a laser spot size of $12 \mu \mathrm{m}$ and electron spot size of $3.2 \mu \mathrm{m}$, the $\mathrm{x}$-ray beam energy will be $12 \mathrm{keV}$ with $1.6 \times 10^{5}$ photons/bunch. The $\mathrm{x}$-ray beam flux will be $1.6 \times 10^{13} \mathrm{ph} / \mathrm{s}$, with an average brilliance of $1.6 \times 10^{14} \mathrm{ph} /\left(\mathrm{s} \mathrm{mm}^{2} \mathrm{mrad}^{2} 0.1 \% \mathrm{BW}\right)$. Even with the reduction due to hourglass effects, these values are sufficiently high as to indicate that a compact Compton source which fulfills these parameters is likely to be very interesting to potential users [4]. These quantities are summarized in Table III for the two different laser spot sizes.

Table IV contains some of the current projects with a compact ICLS design. From this table, it can be seen that the concept we propose is among the best performing of
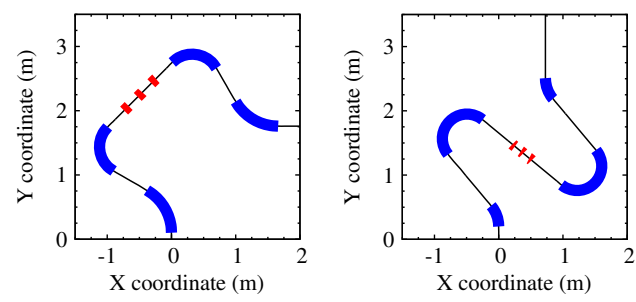

Beam path Quadrupole
Dipole

FIG. 3. Basic layout examples of $3 \pi$ (left) and $4 \pi$ (right) bunch compressors. Beam enters at $(0,0)$. compact ICS light sources. While the total average flux is comparable with many of the listed sources, this concept has a smaller source size and a higher average brilliance in a narrow-bandwidth.

One of the benefits of this design is that the layout is entirely linear, which can be seen in Fig. 1. This benefit allows for a simpler and more compact design. While we have seen improvement in the transverse emittance by increasing the length of the bunch off the cathode, a longer bunch length requires a bunch compressor, increasing both the size and complexity of the design. The bunch compressor might be a $3 \pi$ or $4 \pi$ design, basic examples of which are shown in Fig. 3 [11,37].

\section{SRF GUN}

\section{A. Similar design comparison}

There exist three types of photoinjectors, or guns, presently: the DC gun, the normal conducting rf gun, and the SRF gun. While the first two types represent technology that is mature and the result of development over many decades, SRF guns are still an emerging technology $[2,15]$.

The concept for an SRF gun was initially published in the early 1990s [38], though more consistent publishing on the subject did not occur until nearly a decade later [39-42]. 
TABLE V. Comparison of various gun designs [15,43-45]. All superconducting guns are indicated by (SC) and have a reentrant geometry.

\begin{tabular}{lccccccc}
\hline \hline Parameter & ODU & NPS & WiFEL & BNL & APEX & Cornell & Units \\
\hline Type & SC & SC & SC & SC & NC & DC & \\
Frequency & 500 & 500 & 200 & 112 & 186 & $\mathrm{MHz}$ \\
Bunch charge & 0.01 & 1 & 0.2 & 5 & 0.01 & 0.019 & $\mathrm{nC}$ \\
Transverse normalized rms emittance & 0.11 & 4 & 0.9 & 3 & 0.2 & 0.2 & $\mathrm{~mm} \mathrm{mrad}$ \\
Average current & 1 & 1 & 1 & 50 & $\leq 0.03$ & 26 & $\mathrm{~A}$ \\
\hline \hline
\end{tabular}

Using the idea of a reentrant cavity for an SRF gun was first presented in [39], which subsequently inspired a number of similar gun designs $[15,43]$. Table $\mathrm{V}$ compares various SRF reentrant gun designs with each other and to the parameters ultimately achieved by this study, referred to as ODU in the table. This table contains the design parameters for projects at the Naval Postgraduate School (NPS), the University of Wisconsin FEL (WiFEL), and Brookhaven National Lab (BNL). Two high performing normal temperature guns, the VHF normal conducting rf gun at APEX (APEX) and the Cornell DC gun (Cornell), are also given, for comparison with more established technologies.

There are two considerations that can be seen from Table V. The first is that the bunch charge of the ODU ICLS gun is smaller than the other SRF designs by an order of magnitude or more. The second is that the desired transverse normalized rms emittance is also smaller than the other designs by nearly an order of magnitude or more. This reduced bunch charge is what makes the extremely small emittance feasible. The feasibility of this extremely small emittance for a small bunch charge is demonstrated in the normal temperature guns- the Cornell DC gun in particular has demonstrated comparable emittance and bunch charge [45].

It is common in rf/SRF gun design to mitigate the growth of the transverse emittance of the bunch due to space charge in order to produce a beam with the smallest emittance. Emittance compensation is the reduction of emittance due to linear space-charge forces [46,47]. One of the most common techniques in emittance compensation is the use of a solenoid. By placing a solenoid after an injector, the goal is to manipulate the transverse phase space so that the focusing effect of the solenoid negates the defocusing effect of the space charge [46-48]. This technique is used in the three other SRF gun designs listed in Table V [15].

At the beginning of this study, simulations were run that modeled a bunch exiting the gun which passed through a solenoid before entering the linac. This approach to emittance compensation failed in two ways- the transverse normalized rms emittance was not decreased and the bunch exiting the linac was difficult to manipulate for compression and final focusing [9]. Consequently, in designing the ODU ICLS accelerator a different approach was taken, which utilized rf focusing by altering gun geometry to provide focusing, instead of it being provided by a solenoid as in similar SRF gun designs [15].
Rf focusing refers to focusing provided by the rf EM fields of the accelerating structure [49]. One example of this is shown in [50], where the rf EM fields of the gun are manipulated by recessing the cathode holder by a varying amount. In essence, this alteration to the gun geometry is to produce a radial electric field which focuses the beam. Ideally, the focusing provided will negate the defocusing produced by the space charge. However, there is a cost to this approach. As the cathode is further recessed, the radial component of the electric field (and thus the focusing) increases, but the longitudinal component (which accelerates the beam) decreases [50].

By changing the geometry of the nosecone, it is also possible to alter the EM fields within the gun. Regardless of how the radial field is produced, there is still a balancing act that must be found between the accelerating and focusing fields. Given that increasing the focusing field decreases the accelerating one, a simplistic line of thought leads one to simply increase the operating gradient until the bunch that exits the gun is sufficiently relativistic such that space charge is negligible. There are two main reasons that such an approach is not feasible.

First, for any given gun geometry there is a point at which increasing the operating gradient is more detrimental than beneficial to the beam quality. Past this point, the strength of the focusing field is actually over-compensating for the effects of space charge on the bunch, increasing the emittance at the exit of the linac. Therefore, in general there exists an operating gradient for a given geometry which produces the smallest transverse emittance, analogous to choosing the correct lens focal length to focus a beam of light at a particular location. Second, there exists a maximum threshold for surface fields on an SRF structure for reliable function. As the operating gradient is directly proportional to the surface fields, a maximum threshold for the operating gradient exists for any given geometry [11].

\section{B. Initial bunch distribution and drive laser}

The initial bunch distribution off the cathode has the properties given in Table VI. This bunch is long enough to make longitudinal space charge effects negligible, while short enough to remove the need for a bunch compressor, which simplifies the design [11]. In order to produce a 4.5 psec flat-top bunch off the cathode, there exist multiple options. One fully realized option is in use in the LCLS 
TABLE VI. Bunch distribution off the cathode.

\begin{tabular}{lcc}
\hline \hline Parameter & Quantity & Units \\
\hline Longitudinal distribution & Plateau & \\
Bunch length & 4.5 & $\mathrm{ps}$ \\
Rise time & 1.125 & $\mathrm{ps}$ \\
Radial distribution & Uniform & \\
$r m s$ bunch radius & 1 & $\mathrm{~mm}$ \\
Initial transverse momentum & 0 & $\mathrm{mrad}$ \\
Bunch charge & 10 & $\mathrm{pC}$ \\
Initial kinetic energy & 1 & $\mathrm{keV}$ \\
$p_{z}$ distribution & Isotropic & \\
\hline \hline
\end{tabular}

injector [51]. This drive laser was manufactured by Thales Laser and is a frequency tripled, chirped-pulse amplification system based on a Ti:sapphire laser $[51,52]$. The specifications called for by the LCLS commissioning require a FWHM pulse duration of 6 ps with a repetition rate of up to $120 \mathrm{~Hz}$. In addition, the laser has an adjustable pulse duration between 3 and 20 ps [51]. While the pulse duration is in the correct regime this concept requires, the repetition rate is less than required by nearly two orders of magnitude.

Another scheme for producing a flat-top bunch off the cathode involves the use of long-period fiber gratings (LPGs). Using this approach, it has been demonstrated experimentally that Gaussian-like optical pulses can be transformed into flat-top pulses. In the proof of concept experiment which confirmed this approach, $600 \mathrm{fs}$ and $1.8 \mathrm{ps}$ Gaussian-like pulses were transformed into 1 and 3.2 ps flattop pulses, respectively. The same LPG was used for both transformations, demonstrating the adaptability of such a device [53]. It remains to demonstrate this technology at high average power.

The most comparable laser to the desired specifications is likely a recent $\mathrm{Yb}$-fiber-based, high-rep-rate picosecond source. With a $79.3 \mathrm{MHz}$ repetition rate and a time duration of $20 \mathrm{ps}$, this source is close enough that matching the parameters is a reasonable proposition-especially using the shaping techniques previously touched on to generate the correct distribution off the cathode [54]. Another Ybfiber-based, high-rep-rate source also exists, though the generated pulses are in the sub-picosecond range [55]. There do exist non-Gaussian, high-rep-rate Fabry-Pérot lasers, though the mismatch in desired parameters may mean that using another type of drive laser may be a simpler approach [56,57].

\section{Optimization leading to the geometry}

During the course of the design further gun optimization was necessary to obtain the desired electron beam at the IP. To support the optimization it was necessary to create a set of parameters to fully define the parametric piecewise function that describes the gun shape, assuming the overall gun shape is retained. A set of formulas was created that required twelve parameters, shown in Fig. 4.

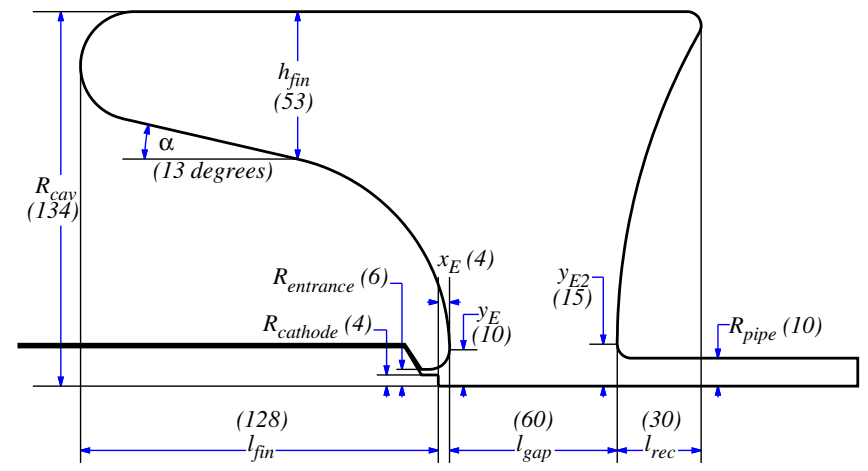

FIG. 4. Diagram of gun geometry with parameters and quantities. Quantities are rounded to the nearest millimeter and given in millimeters, except where explicitly indicated.

While a cursory examination was made of different parameters, $y_{E}$ is the key parameter to change to produce a suitable electron beam at the interaction point. This parameter being key is not surprising, given its proximity to both the center of the gun and the cathode holder. By altering this parameter over a range of values and evaluating the electron beam at the exit of the linac, the gun geometry was chosen, with $y_{E}=10 \mathrm{~mm}$. Further optimization of the other parameters may produce a better design at a later date.

\section{Final geometry and simulation results}

The optimized geometry is shown in the top left of Fig. 1, with the physical and rf properties given in Table VII.

TABLE VII. Cavity and rf properties of the gun design. Set to operate at $E_{\mathrm{acc}}=10.3 \mathrm{MV} / \mathrm{m}$.

\begin{tabular}{|c|c|c|}
\hline Parameter & Quantity & Units \\
\hline Frequency of accelerating mode & 500 & $\mathrm{MHz}$ \\
\hline Cavity length & 221.5 & $\mathrm{~mm}$ \\
\hline Cavity radius & 134 & $\mathrm{~mm}$ \\
\hline Cavity gap & 69 & $\mathrm{~mm}$ \\
\hline Beamport aperture radius & 10 & $\mathrm{~mm}$ \\
\hline Peak electric surface field $E_{p} / E_{\text {acc }}$ & 3.86 & \\
\hline Peak magnetic surface field $B_{p} / E_{\text {acc }}$ & 6.55 & $\mathrm{mT} /(\mathrm{MV} / \mathrm{m})$ \\
\hline$B_{p} / E_{p}$ & 1.70 & $\mathrm{mT} /(\mathrm{MV} / \mathrm{m})$ \\
\hline Geometrical factor, $G$ & 83.7 & $\Omega$ \\
\hline$(R / Q) \times G$ & $1.31 \times 10^{4}$ & $\Omega^{2}$ \\
\hline Energy content $^{\mathrm{a}}$ & 44 & $\mathrm{~mJ}$ \\
\hline
\end{tabular}

${ }^{\mathrm{a}}$ At $E_{\mathrm{acc}}=1 \mathrm{MV} / \mathrm{m}$.

TABLE VIII. IMPACT-T tracking results at gun exit.

\begin{tabular}{lcc}
\hline \hline Parameter & Quantity & Units \\
\hline Kinetic energy & 1.51 & $\mathrm{MeV}$ \\
$r m s$ energy spread & 0.68 & $\mathrm{keV}$ \\
$\sigma_{x, y}$ & 0.29 & $\mathrm{~mm}$ \\
$\epsilon_{(x, y), \mathrm{rms}}^{N}$ & 0.20 & $\mathrm{~mm} \mathrm{mrad}$ \\
$\sigma_{z}$ & 0.18 & $\mathrm{~mm}$ \\
\hline \hline
\end{tabular}



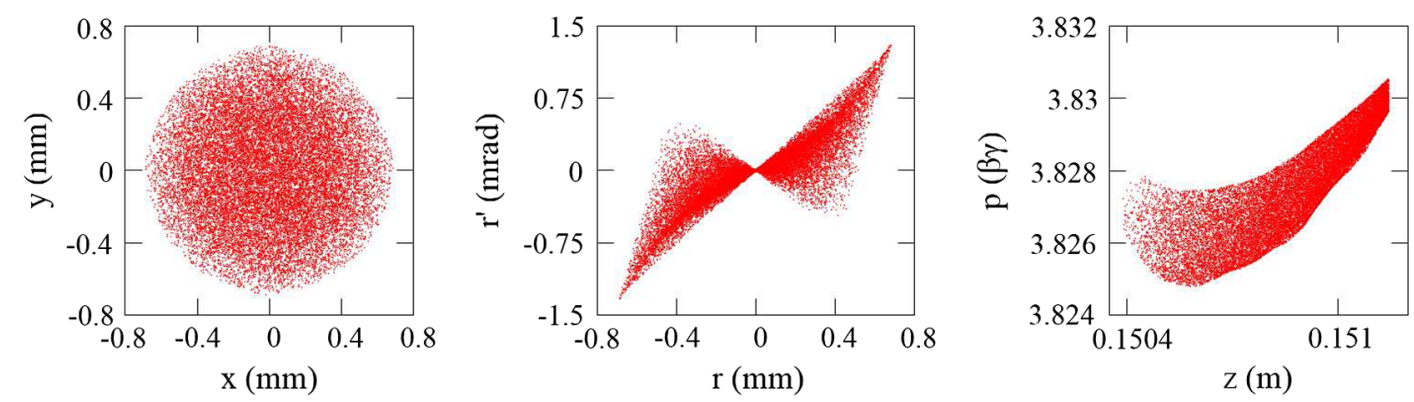

FIG. 5. Beam spot (left), transverse phase space (center), and longitudinal phase space (left) of bunch exiting gun.

We used IMPACT-T [58] to track 100000 macroparticles through the EM fields simulated by SUPERFISH [59]. The tracking results at the exit of the gun are shown in Table VIII, with the transverse phase space and beam spot at the gun exit shown in Fig. 5.

\section{LINAC}

\section{A. SRF double-spoke cavity}

Until recently, accelerating electrons near the speed of light has not been attempted with multispoke cavities. This is largely because of the well-established and successful performance of TM-type cavities. However, multispoke cavities are familiar options for accelerating ions. Previous studies of multispoke cavities for $\beta \sim 1$ strongly suggest that they are a viable option for accelerating electrons [20,60-63]. Compared to typical TM cavities, spoke cavities of the same frequency have smaller diameters and are easier to operate at $4.2 \mathrm{~K}$. Additionally, the fundamental mode of multispoke cavities for speed-oflight beams is the lowest frequency mode, simplifying damping and extraction of higher-order modes. Overall, multispoke cavities are particularly well-suited for lowfrequency applications at $4.2 \mathrm{~K}$ [64].

The four $500 \mathrm{MHz}$ cavities which comprise the linac are double-spoke speed-of-light SRF cavities designed by Christopher Hopper during his ODU $\mathrm{PhD}$ research $[25,64,65]$. The electron beam gains nearly $5.9 \mathrm{MeV}$ as

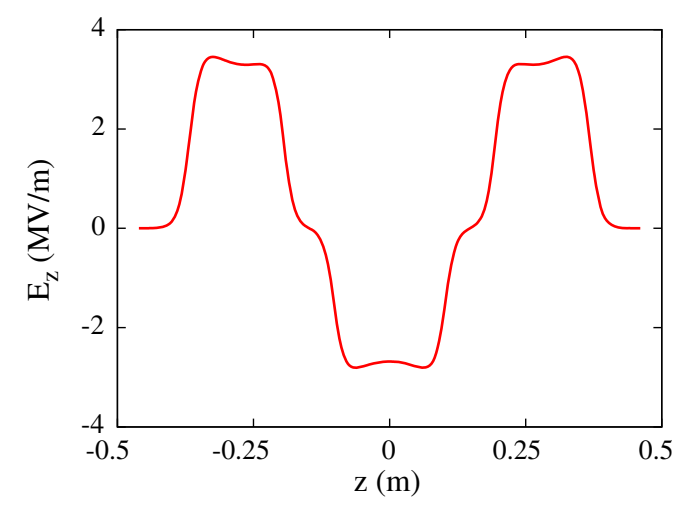

FIG. 6. The accelerating electric field along the beamline of the double-spoke SRF cavity. it passes through each cavity in the linac. An image of this cavity, with a portion cut away to more clearly view the interior structure, is shown in the bottom right of Fig. 1. The accelerating field of this cavity is shown in Fig. 6, with the complete EM field calculated by CST MICROWAVE STUDIO® [66]. Select rf and physical properties are contained in Table IX. For more information on the optimization of the double-spoke cavity design, the reader is directed toward [64]. The linac is designed to be as short as possible-both the number of cavities and separation between cavities are minimized. To decrease the number of cavities while still accelerating the electron beam to $25 \mathrm{MeV}$ would require the three cavities to operate at unreliably high gradients. The current separation between SRF structures is only what is necessary for cavities within the same or adjacent cryomodules.

\section{B. Layout and simulation results}

One aspect of the double-spoke cavity is the "quadrupole-like" behavior of the cavities - the electron beam is focused in $x$ and defocused in $y$, or vice versa by

TABLE IX. Cavity and rf properties of double-spoke cavity.

\begin{tabular}{lcc}
\hline \hline Parameter & Quantity & Units \\
\hline Frequency of accelerating mode & 500 & $\mathrm{MHz}$ \\
Frequency of nearest mode & 507.1 & $\mathrm{MHz}$ \\
Cavity diameter & 416.4 & $\mathrm{~mm}$ \\
Iris-to-iris length & 725 & $\mathrm{~mm}$ \\
Cavity length & 805 & $\mathrm{~mm}$ \\
Reference length $\left[(3 / 2) \beta_{0} \lambda\right]$ & 900 & $\mathrm{~mm}$ \\
Aperture diameter & 50 & $\mathrm{~mm}$ \\
Energy gain ${ }^{\mathrm{a}}$ at $\beta_{0}$ & 900 & $\mathrm{kV}$ \\
$R / Q$ & 675 & $\Omega$ \\
$Q R_{s}^{\mathrm{b}}$ & 174 & $\Omega$ \\
$(R / Q) \times Q R_{s}^{\mathrm{b}}$ & $1.2 \times 10^{5}$ & $\Omega^{2}$ \\
Peak electric surface field $E_{p} / E_{\text {acc }}$ & 3.7 & \\
Peak magnetic surface field $B_{p} / E_{\mathrm{acc}}$ & 7.6 & $\mathrm{mT} /(\mathrm{MV} / \mathrm{m})$ \\
$B_{p} / E_{p}$ & 2.05 & $\mathrm{mT} /(\mathrm{MV} / \mathrm{m})$ \\
Energy content $^{\mathrm{a}}$ & 0.38 & $\mathrm{~J}$ \\
Power dissipation & 0.87 & $\mathrm{~W}$ \\
\hline \hline
\end{tabular}

\footnotetext{
${ }^{\mathrm{a}}$ At $E_{\text {acc }}=1 \mathrm{MV} / \mathrm{m}$ and reference length $(3 / 2) \beta_{0} \lambda, \beta_{0}=1$. ${ }^{\mathrm{b}} R_{s}=125 \mathrm{n} \Omega$.
} 
TABLE X. Properties of electron bunch at linac exit.

\begin{tabular}{lcc}
\hline \hline Parameter & Quantity & Units \\
\hline Kinetic energy & 25. & $\mathrm{MeV}$ \\
$r m s$ energy spread & 3.44 & $\mathrm{keV}$ \\
$\epsilon_{x, \mathrm{rms}}^{N}$ & 0.10 & $\mathrm{~mm} \mathrm{mrad}$ \\
$\epsilon_{y, \mathrm{rms}}^{N}$ & 0.13 & $\mathrm{~mm} \mathrm{mrad}$ \\
$\sigma_{x}$ & 0.35 & $\mathrm{~mm}$ \\
$\sigma_{y}$ & 0.38 & $\mathrm{~mm}$ \\
$\beta_{x}$ & 60 & $\mathrm{~m}$ \\
$\beta_{y}$ & 54 & $\mathrm{~m}$ \\
$\alpha_{x}$ & -2.3 & \\
$\alpha_{y}$ & -3.8 & $\mathrm{~mm}$ \\
$\sigma_{z}$ & 0.67 & \\
\hline \hline
\end{tabular}

the accelerating mode $[13,25,67]$. This aspect means that some adjustment is necessary to provide a round beam spot to the bunch compressor or final focusing section. When arranging the double-spoke cavities, the center two cavities are rotated $180^{\circ}$ around the $y$-axis, as seen in Fig. 1 . Simulations have demonstrated that this layout produces the roundest beam at the exit of the linac.

Continuing to simulate the beam past the gun exit yields the electron beam properties given in Table $\mathrm{X}$ with the beam spot and phase spaces shown in Fig. 7. The final two cavities are chirped $-4^{\circ}$ off-crest in order to reduce the rms energy spread. At this location, the extremely small transverse normalized rms emittance has been achieved.
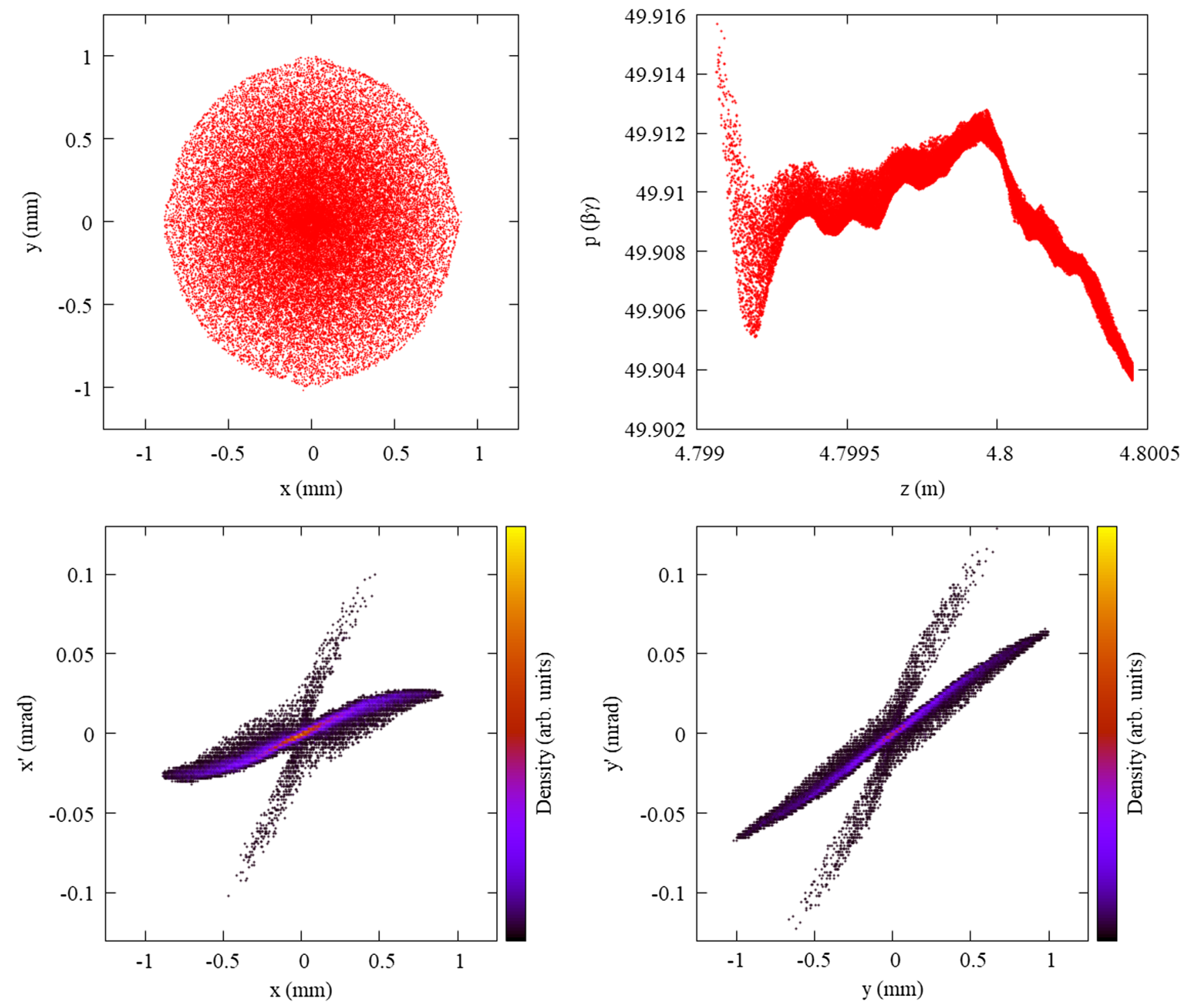

FIG. 7. Beam spot (upper left), longitudinal phase space (upper right), horizontal phase space (bottom left), and vertical phase space (bottom right) of bunch after exiting the linac. 


\section{Emittance decrease}

It has been noted before that the transverse normalized rms emittance of the bunch out of the gun is not necessarily the same out of the linac. In the first iteration of the gun design, there was an increase in emittance after the bunch exited the gun because it was not yet at a sufficient energy to make space charge negligible. In the final design, however, the emittance actually decreases between the gun and linac exits. The final iteration has a greater decrease in emittance and will be examined here to explain the behavior.

The transverse normalized rms emittances and $r m s$ spot sizes of the bunch as it passes through the linac are shown in Fig. 8. Both horizontal and vertical emittances decrease through the linac, though the rate of decrease changes with the longitudinal position and which transverse component is being considered. The transverse rms sizes of the beam grow rapidly immediately after the bunch exits the gun, but the size increase is limited within the linac.

Using IMPACT-T, it is possible to see the evolution of the bunch after the gun as the beam drifts downstream, without passing through the linac. The transverse normalized rms emittance and the spot size of such a drifting bunch are shown as a function of longitudinal position in Fig. 9. While the spot size increases as the bunch drifts
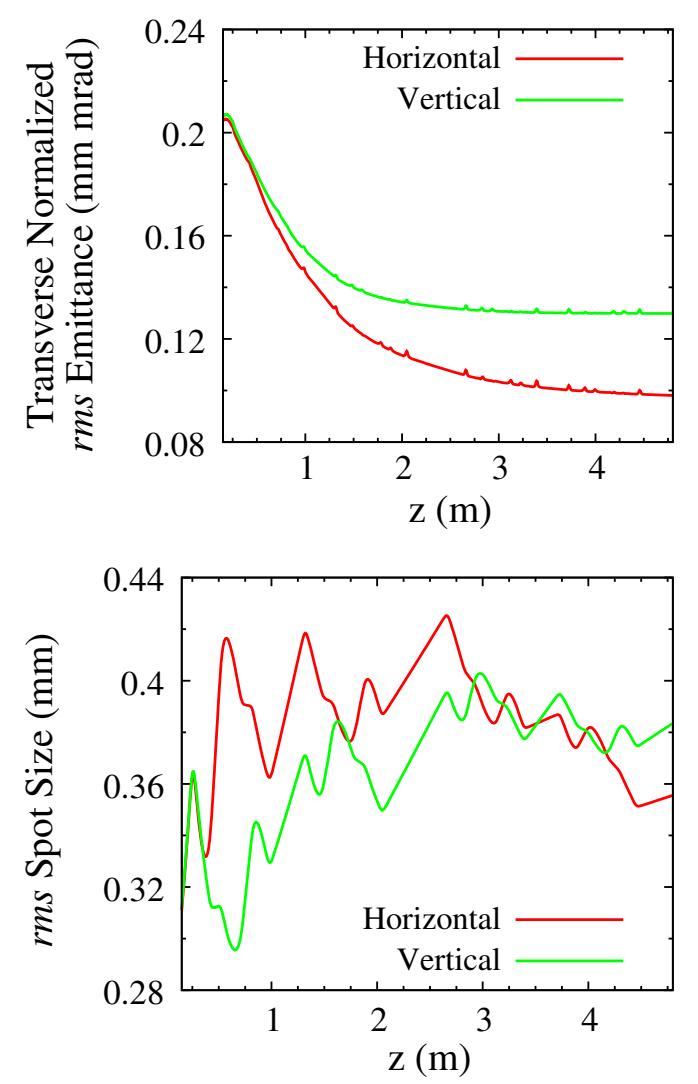

FIG. 8. Transverse normalized rms emittances (top) and spot sizes (bottom) of bunch passing through the linac.
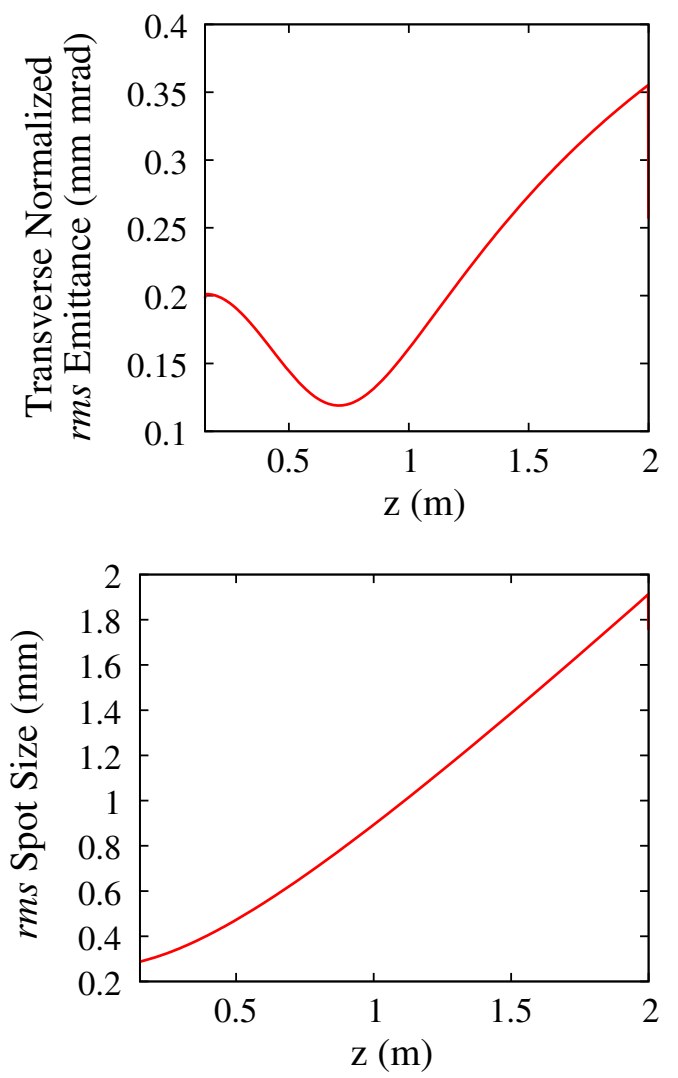

FIG. 9. Transverse normalized $r m s$ radial emittance (top) and transverse spot size (bottom) of bunch drifting after gun exit as a function of longitudinal position.

downstream, the emittance decreases to a minimum at approximately $z=0.7 \mathrm{~m}$, before increasing — an emittance rebound.

One further aspect of interest is that for the drifting bunch, $\epsilon_{r, \mathrm{rms}}^{N}=0.12 \mathrm{mmmrad}$ at the minimum of $z=0.7 \mathrm{~m}$, but at the exit of the linac $\epsilon_{x, \mathrm{rms}}^{N}=0.10 \mathrm{~mm} \mathrm{mrad}$ and $\epsilon_{y, \mathrm{rms}}^{N}=0.13 \mathrm{~mm} \mathrm{mrad}$. So even the average of the two transverse emittances is less than what can be attained if the bunch just drifts after the gun. If the bunch charge of the beam exiting the gun is artificially decreased, the distance to the emittance minimum increases and the emittance minimum decreases. This can be considered analogous to increasing the beam energy without the additional phase space manipulations of passing the beam through the "quadrupole-like" spoke cavities.

Increasing the energy of the beam does not mean it is impossible for an emittance rebound to occur in the linac; it depends on the bunch exiting the gun. One example of such an emittance rebound is shown in Fig. 10. The figure shows the transverse normalized rms emittances of the cathode bunch tracked through an unoptimized version of the accelerating section. While the emittances decrease, after the minimum both increase. At this minimum, $\epsilon_{x, \mathrm{rms}}^{N}=0.095 \mathrm{~mm} \mathrm{mrad}$ and $\epsilon_{y, \mathrm{rms}}^{N}=0.11 \mathrm{~mm} \mathrm{mrad}$, both 


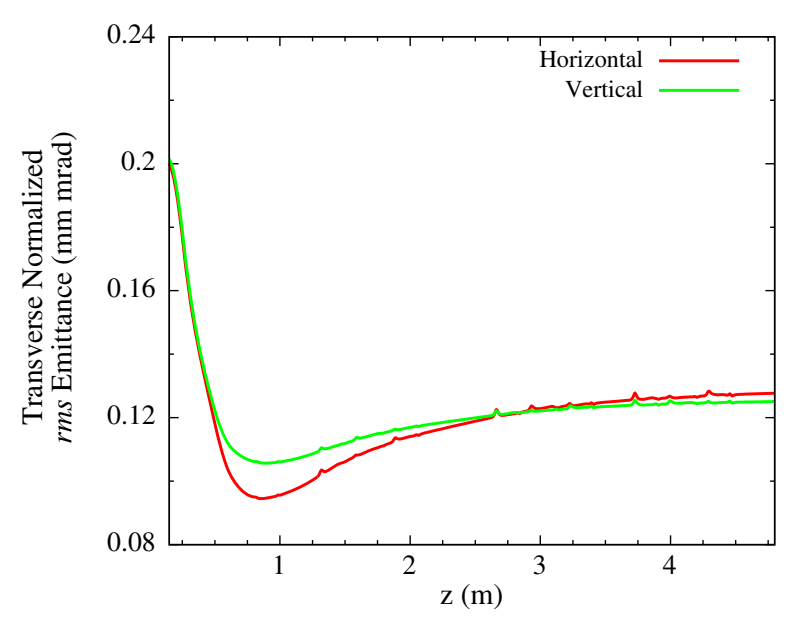

FIG. 10. Transverse normalized rms emittances of the bunch off the cathode tracked through an unoptimized version of the accelerating section as a function of the longitudinal position.

of which are smaller values, respectively, than those of the bunch exiting the linac. With the increase after the minimum, the bunch exits with $\epsilon_{x, \mathrm{rms}}^{N}=0.13 \mathrm{~mm}$ mrad and $\epsilon_{y, \mathrm{rms}}^{N}=0.13 \mathrm{mmmrad}$, so this is not the best possible system for this initial bunch. Consequently, there is some limit on the rate of emittance decrease for the bunch exiting the gun. If the emittance decreases too rapidly, an emittance rebound occurs in the linac, which leads to the beam quality suffering.

The driving characteristic of the emittance behavior in the linac is the emittance evolution of the bunch as it exits the gun, not the emittance value itself. For a given geometry, two different operating gradients of the gun can produce the same emittance value at the gun exit, but only one may lead to an emittance rebound. Consequently, tracking the beam through to the end of the linac is necessary for appropriate evaluation of the gun geometry and settings.

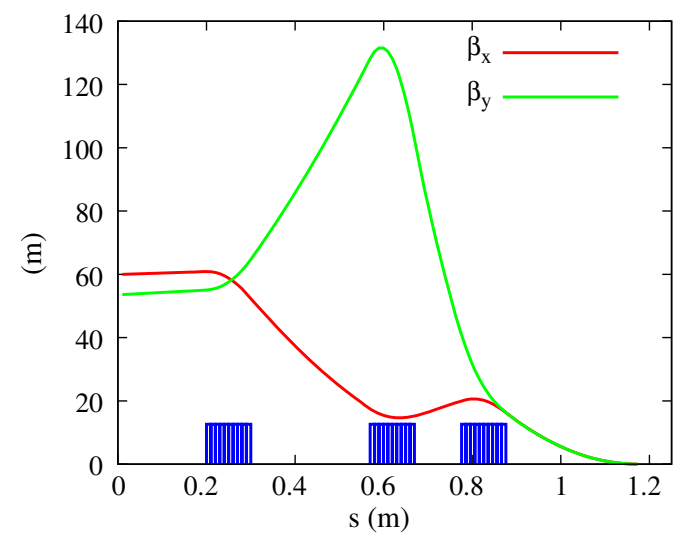

FIG. 11. $\beta_{x}$ and $\beta_{y}$ as a function of $s$ in the final focusing section of the design. The locations of the three quadrupoles are shown along the horizontal axis.
TABLE XI. Select magnet properties of the final focusing section.

\begin{tabular}{lcc}
\hline \hline Parameter & Quantity & Units \\
\hline Maximum $\beta$ & 132 & $\mathrm{~m}$ \\
Quadrupole length & 0.1 & $\mathrm{~m}$ \\
Quadrupole strengths & $1.2-3.6$ & $\mathrm{~T} / \mathrm{m}$ \\
$r m s$ energy spread & 3.4 & $\mathrm{keV}$ \\
\hline \hline
\end{tabular}

\section{FINAL FOCUSING}

The final focusing section consists of three quadrupoles, with a distance of $\sim 29 \mathrm{~cm}$ between the third quadrupole and the IP. Tracking was performed using Elegant and the bunch distribution at the exit of the linac [68]. Elegant was used in order to make the optimization easier, but the results were later compared to tracking using a 3D space charge tracking code and found to have negligible differences. Aberrations of quadrupole displacement were included in the sensitivity studies. The value of $\beta_{x}$ and $\beta_{y}$ are shown as a function of the beam path $s$ in Fig. 11. Certain aspects of the focusing lattice and the properties of the bunch at the IP are shown in Tables XI and XII, respectively. The beam spot and phase spaces of this beam are shown in Fig. 12. It can be seen that the small emittance is preserved while focusing the electron beam spot size to a few microns.

There is an assumption that the use of spoke cavities in the linac instead of traditional TM-type cavities, such as multicell elliptical cavities, has a detrimental effect on the transverse emittance of the beam. In order to examine this idea, each double-spoke cavity in the linac was replaced by a 3-cell elliptical cavity; the EM fields were calculated using SUPERFISH. After using IMPACT-T to track the bunch through this version of the linac, the beam was focused to a small size. The beam spot and phase spaces of the focused bunch is shown in Fig. 13. When compared to the bunch accelerated by the double-spoke cavities, seen in Fig. 12, the beam is highly comparable. The transverse normalized rms emittance of the new bunch is $\sim 0.11 \mathrm{mmmrad}$, which is the average transverse

TABLE XII. Select properties of the electron beam parameters, both desired and achieved, at the IP.

\begin{tabular}{lccc}
\hline \hline Parameter & Desired & Achieved & Units \\
\hline$\beta_{x}$ & 5 & 5.4 & $\mathrm{~mm}$ \\
$\beta_{y}$ & 5 & 5.4 & $\mathrm{~mm}$ \\
$\epsilon_{x, \mathrm{rms}}^{N}$ & 0.1 & 0.1 & $\mathrm{~mm} \mathrm{mrad}$ \\
$\epsilon_{y, \mathrm{rms}}^{N}$ & 0.1 & 0.13 & $\mathrm{~mm} \mathrm{mrad}$ \\
$\sigma_{x}$ & 3.2 & 3.4 & $\mu \mathrm{m}$ \\
$\sigma_{y}$ & 3.2 & 3.8 & $\mu \mathrm{m}$ \\
$>76 \%$ longitudinal distribution & 3 & 3 & $\mathrm{ps}$ \\
$r m s$ energy spread & 7.5 & 3.4 & $\mathrm{keV}$ \\
\hline \hline
\end{tabular}



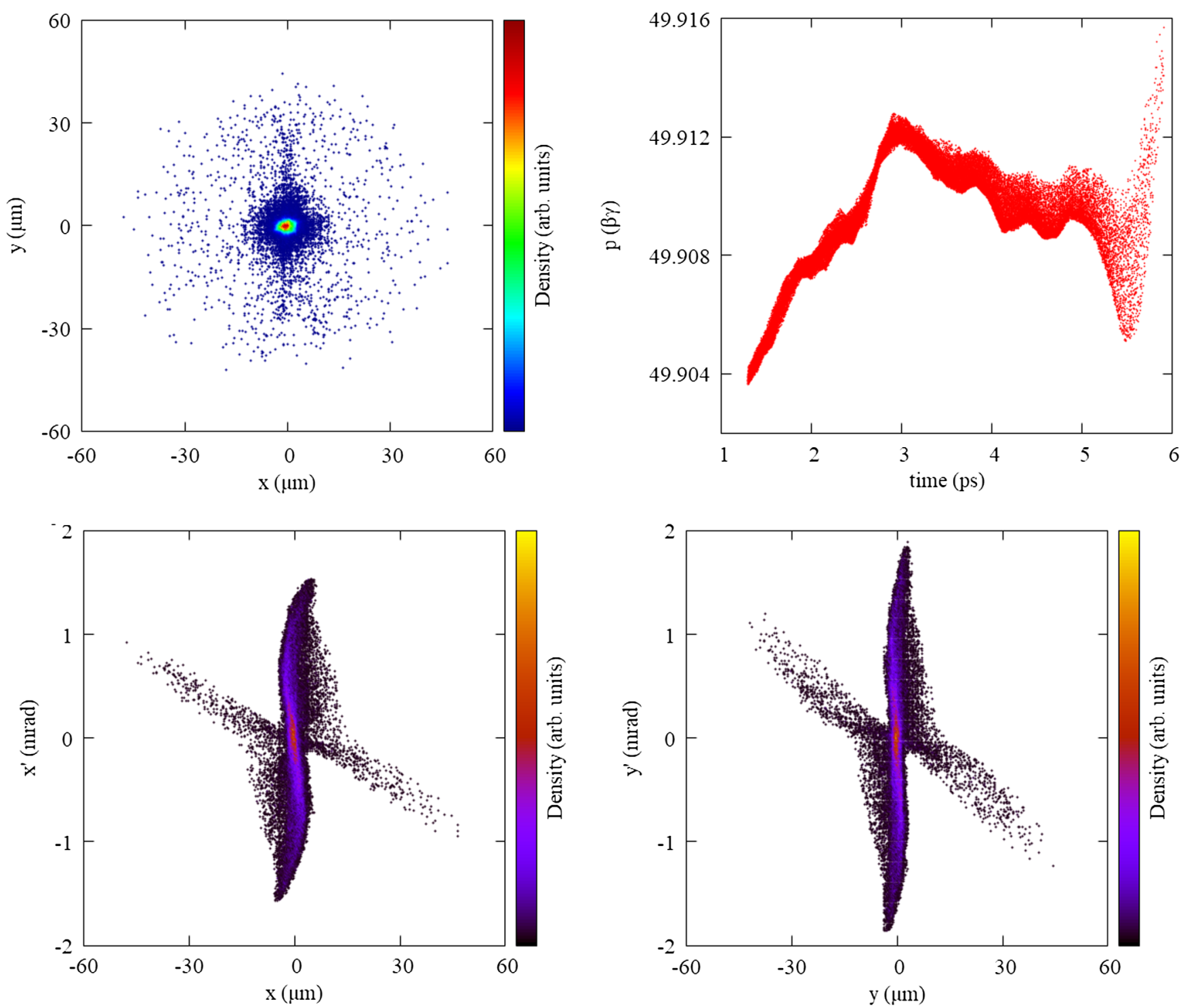

FIG. 12. Beam spot (top left), longitudinal phase space (top right), horizontal phase space (bottom left), and vertical phase space (bottom right) of the electron bunch at the IP.

normalized rms emittance of the bunch using spoke cavities. Consequently, there is no beam physics reason that elliptical cavities are the better option for beam acceleration.

\section{SENSITIVITY STUDIES}

In order to ascertain the robustness of the design, simulations involving deviations from optimal design parameters were performed. In these simulations, the maximum threshold for each perturbation from the design was determined to be the point when any electron beam parameter value given in Table XI changed by $20 \%$.

The phase and amplitude of each SRF structure was individually varied while holding all other settings constant. The change in phase is given in degrees, while the change in amplitude is given in percentage of the design value. The thresholds are reported in Table XIII. The limiting electron beam parameter is the vertical rms size when the amplitude of the cavities is varied. In all other cases, the limiting parameter is the rms energy spread.

Systematic perturbations were also evaluated for the coordinates of both the linac cavities and the magnets in the final focusing section, separately. In either case, each element was randomly attributed some amount of misalignment in each of the three Cartesian directions. The maximum possible misalignment is the threshold. For the translational (transverse and longitudinal) misalignment in the linac cavities, the threshold is $500 \mu \mathrm{m}$, with the limiting electron beam parameter being the rms energy spread. For the translational misalignment of the three quadrupole magnets, the threshold is $300 \mu \mathrm{m}$, with the limiting parameter being the vertical size [11]. 

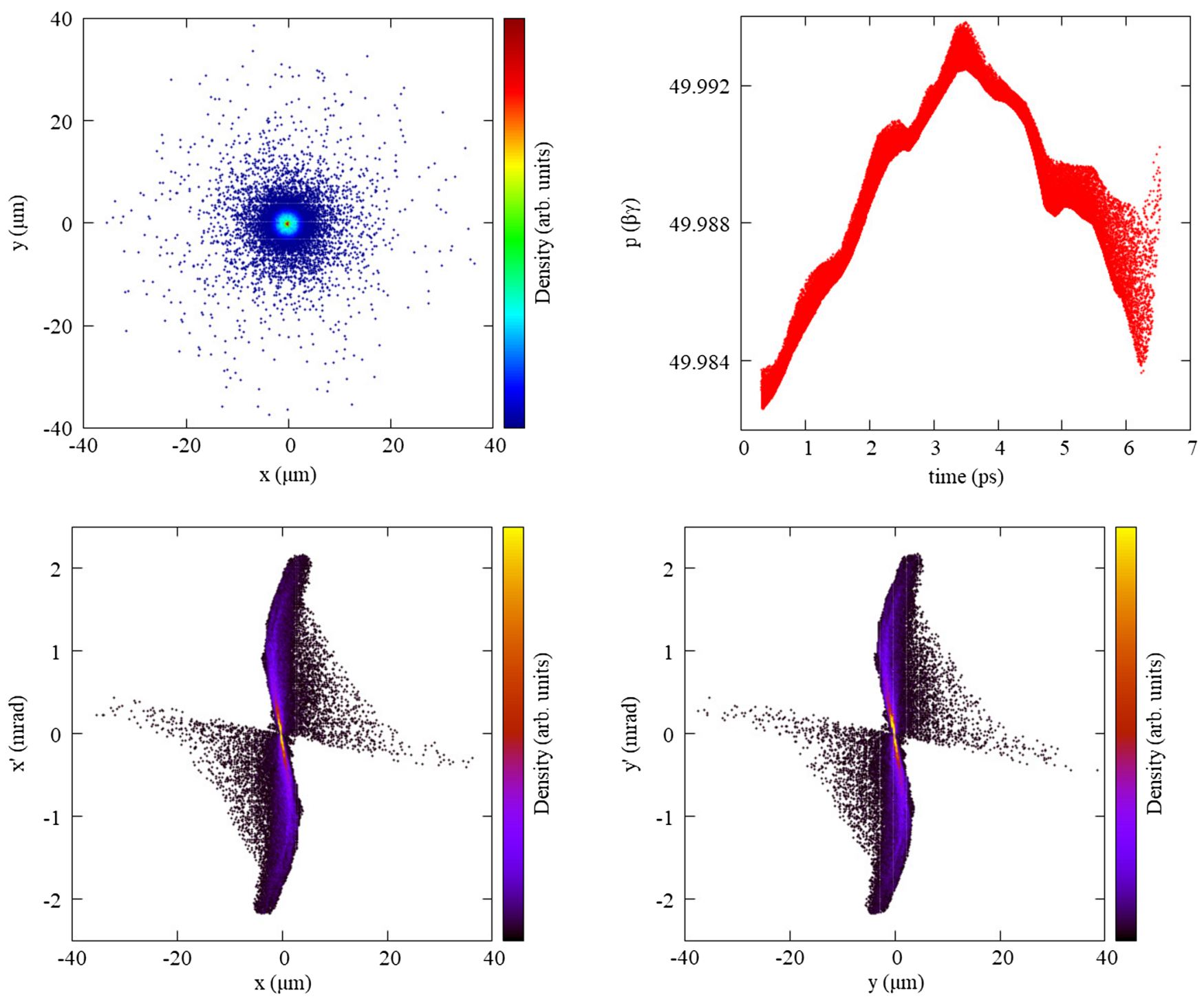

FIG. 13. Beam spot (top left), longitudinal phase space (top right), horizontal phase space (bottom left) and vertical phase space (bottom right) of the electron bunch at the IP, using elliptical cavities in the linac.

\section{INCIDENT LASER}

TABLE XIII. The amplitude and phase perturbation from design for each SRF structure at which some electron beam parameter changes $\sim 20 \%$ at the IP.

\begin{tabular}{lc}
\hline \hline Varied Parameter and Structure & Threshold \\
\hline Amplitude of Gun & $-2.0 \%$ \\
& $+0.6 \%$ \\
Amplitude of All Cavities & $-1.0 \%$ \\
& $+0.8 \%$ \\
Phase of Gun & $-7.2^{\circ}$ \\
& $+1.2^{\circ}$ \\
Phase of All Cavities & $-1.2^{\circ}$ \\
& $+1.2^{\circ}$ \\
\hline \hline
\end{tabular}

Inverse Compton Light Sources require both an electron beam and an incident laser, the latter of which has been neglected thus far. The design of the appropriate laser is beyond the scope of this article, but the desired properties are provided in Table II. While a laser with a circulating power of $1 \mathrm{MW}$ is called for, such a laser does not currently exist. Current consensus of those within that field is that such a laser is feasible, but until now there has not been a need for it to be developed. At present, high average power lasers currently constructed have a power of $\sim 100 \mathrm{~kW}$. Using a laser with this circulating power would decrease the flux and brightness of the anticipated $x$-ray beam by an order of magnitude. 
TABLE XIV. Estimated x-ray performance assuming design electron beam attained at IP, compared to desired parameters.

\begin{tabular}{|c|c|c|c|c|c|}
\hline \multirow{3}{*}{ Parameter } & \multicolumn{4}{|c|}{ Laser Spot Size $(\mu \mathrm{m})$} & \multirow[b]{2}{*}{ Units } \\
\hline & \multicolumn{2}{|c|}{3.2} & \multicolumn{2}{|c|}{12} & \\
\hline & Desired & Achieved & Desired & Achieved & \\
\hline X-ray energy & 12 & 12 & 12 & 12 & $\mathrm{keV}$ \\
\hline Photons/bunch & $2.4 \times 10^{5}$ & $2.2 \times 10^{5}$ & $1.6 \times 10^{5}$ & $1.6 \times 10^{5}$ & \\
\hline Flux & $2.4 \times 10^{13}$ & $2.2 \times 10^{13}$ & $1.6 \times 10^{13}$ & $1.6 \times 10^{13}$ & $\mathrm{ph} / \mathrm{s}$ \\
\hline Flux in $0.1 \% \mathrm{BW}$ & $3.6 \times 10^{10}$ & $3.3 \times 10^{10}$ & $2.4 \times 10^{10}$ & $2.4 \times 10^{10}$ & $\mathrm{ph} /(\mathrm{s} 0.1 \% \mathrm{BW})$ \\
\hline Average brilliance & $4.4 \times 10^{14}$ & $3.4 \times 10^{14}$ & $1.6 \times 10^{14}$ & $1.2 \times 10^{14}$ & $\mathrm{ph} /\left(\mathrm{s} \mathrm{mm}^{2} \mathrm{mrad}^{2} 0.1 \% \mathrm{BW}\right)$ \\
\hline
\end{tabular}

TABLE XV. X-ray performance of the designs attained by numerical simulation with an aperture of $1 / 40 \gamma$. Suggested aperture for brilliance calculation only.

\begin{tabular}{lccc}
\hline \hline & \multicolumn{2}{c}{ Laser Spot $(\mu \mathrm{m})$} & \\
\cline { 2 - 3 } Parameter & 3.2 & 12 & Units \\
\hline X-ray energy & 12.3 & 12.3 & $\mathrm{keV}$ \\
$N_{0.1 \%}$ & 1230 & 92.4 & $\mathrm{ph} / 0.1 \% \mathrm{BW}$ \\
$\mathcal{S}_{0.1 \%}$ & $1.23 \times 10^{11}$ & $9.24 \times 10^{9}$ & $\mathrm{ph} /(\mathrm{s} 0.1 \% \mathrm{BW})$ \\
$\mathcal{B}_{p}$ 贷 & $4.61 \times 10^{15}$ & $1.58 \times 10^{14}$ & $\mathrm{ph} /\left(\mathrm{s} \mathrm{mm} \mathrm{mrad}^{2} 0.1 \% \mathrm{BW}\right)$ \\
\hline \hline
\end{tabular}

\section{X-RAY SOURCE}

By using the parameter values in Tables XI and II in the formulas presented in Secs. VIII and II A, it is possible to estimate the X-ray beam parameters of the light source. These parameters are presented in Table XIV, assuming Gaussian laser and beam spots, taking into account the reduction due to hourglass effects. This reduction has altered slightly, due to the different electron beam properties at the IP. However, the electron distribution at the interaction point is not Gaussian, bringing the validity of the results into question.

Fortunately, Compton scattering calculations have been made recently which utilize the electron beam

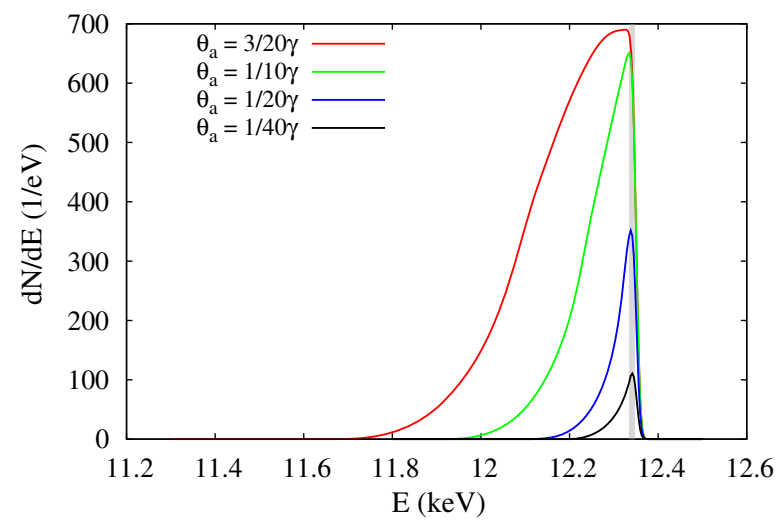

distribution, not just beam parameters. Using ICCS (Improved Codes for Compton Simulation) [12], the calculations of the x-ray light source parameters verify that the non-Gaussian distribution does not significantly impact the anticipated brilliance [10]. ICCS is a simulation code which computes the spectra of interaction between electron beams and scattering laser [12]. Following the same reasoning given for the brilliance formula in Sec. II A, the typical pin-hole brilliance of the x-ray beam $\mathcal{B}_{p}$ becomes

$$
\mathcal{B}_{p}=\lim _{\theta_{a} \rightarrow 0} \frac{\mathcal{S}_{0.1 \%}}{2 \pi^{2} \sigma_{e}^{2} \theta_{a}^{2}}
$$

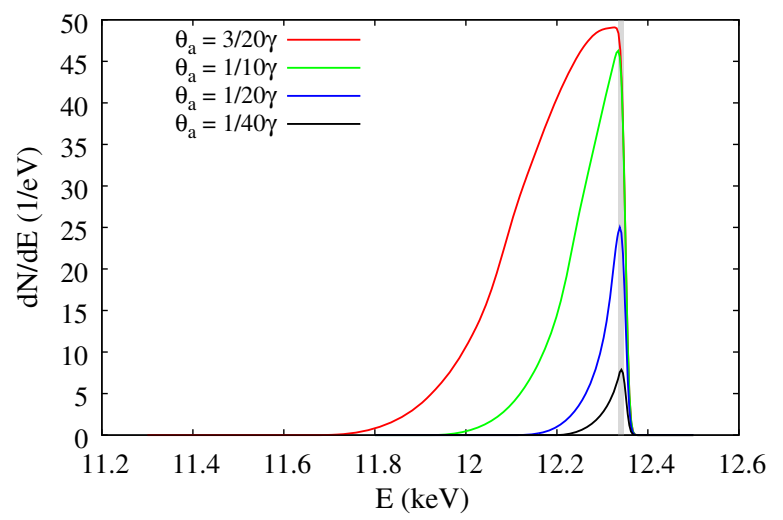

FIG. 14. Number spectra for different apertures generated using 4000 particles for a $3.2 \mu \mathrm{m}$ laser spot size (left) and a $12 \mu \mathrm{m}$ laser spot size (right). Grey box indicates $0.1 \%$ bandwidth. Suggested apertures for brilliance calculation only. 
where $\mathcal{S}_{0.1 \%}$ is the number of photons per second in a $0.1 \%$ bandwidth transmitted through the aperture, which is calculated using ICCS. Table XV contains the estimated $\mathrm{X}$-ray beam parameters when calculated in this manner, while the full spectrum of the produced x-rays is shown in Fig. 14.

The energy and average brilliance in Tables XIV and XV show excellent agreement for the $12 \mu \mathrm{m}$ case. The flux into a $0.1 \%$ bandwidth does not, which is expected because this parameter is dependent on the aperture angle of the interaction enclosure. Calculation results clearly demonstrate that the flux increases with the aperture angle, and the results are being reported for a small opening. The factor of two increase between the calculated brilliance and the pin-hole brilliance for the $3.2 \mu \mathrm{m}$ case is expected-the calculation code makes the assumption that every photon of the scattering laser sees the same scattering potential, which is most valid when the laser spot size is much greater than the electron beam spot size. As that assumption is not valid for the $3.2 \mu \mathrm{m}$ case, the code overestimates the anticipated pin-hole brilliance.

\section{FURTHER WORK}

While we have presented here a preliminary design for a high-brilliance, high-flux inverse Compton light source much work remains to be done (analytical, numerical simulation, and experimental) before such a source could be built. In particular, it may be that the choices of frequency and geometry are not optimal and may be revised after further study or advances is the SRF technology. In this section we outline the major items that would require further $R \& D$ activities.

\section{A. SRF Gun}

A number of simulation studies need to be conducted before the SRF gun is built and commissioned-primarily multipacting and mechanical. If geometry alterations are deemed necessary, another optimization based on simulation beam dynamics results may be necessary, including these studies.

Once these studies are completed and the geometry is finalized, the gun will need to be built and commissioned. At this point, experiments can be performed to demonstrate that the transverse normalized rms emittance behavior as the beam drifts after the gun is as expected, supporting the idea that appropriate gun geometry can provide all necessary emittance compensation.

Integration of a photo-cathode and an SRF gun presents a technical challenge which is under investigation in a number of institutions.

\section{B. Beam physics}

Further sensitivity studies are called for, especially if the gun geometry needs to be altered to avoid significant obstacles in multipacting, mechanical stability, or thermal breakdown. Additionally, simulations examining potential wakefields are desired. All these simulations will need to include potential deviations from an ideal design (physical misalignment, errors of amplitude and phase, etc).

\section{SRF linac}

The design presented here is based on a particular choice of frequency and geometry in order to achieve a balance between size (capital cost) and operating cost. This choice may not be optimal and, in particular, the operating cost would still be higher than desired based on the surface resistance assumed in Table IX. However recent progress in SRF R\&D shows potential for a substantial reduction of power dissipation in SRF cavities. Nitrogen doping [69] and infusion [70] during heat treatment have shown large reduction of those losses at $2 \mathrm{~K}$ and higher frequency. Achieving similar results at $4.2 \mathrm{~K}$ and $500 \mathrm{MHz}$ would validate our choices. On a longer term, $\mathrm{Nb}_{3} \mathrm{Sn}$ [71] could offer dramatic reduction in cryogenic losses at $4.2 \mathrm{~K}$ and would even allow operation at higher frequency; such an advance would lead us to revisit our choices as multi-cell TM-type cavities operating around $650 \mathrm{MHz}$ would be attractive. They may even be able to operate without a refrigerator, using instead cryocoolers [72] if vibrations that such systems often generated can be managed.

Eventually, several prototype cavities will need to be built and tested, and all the processes needed to achieve performance (chemistry, heat treatment, cleaning, etc) will have to be developed and demonstrated.

Performance of this light source is contingent upon achieving and maintaining a very small emittance. This will put challenging constraints on the design of the cryomodule and the low level rf control system.

\section{Incident laser}

As previously mentioned, a laser with the desired properties of either spot size does not currently exist. While current technology might suffice in providing an $\mathrm{x}$-ray beam at least as good as any other compact ICLS, this design has the capacity to surpass that threshold. Consequently, such a laser must be designed and commissioned, before the concept presented here is commissioned.

The benefits of developing a better incident laser do not stop with this concept, however. Compact ICLS projects, proposed or existing, can see an improvement in the quality of the x-ray beam they deliver. This approach may be one method which will see such development funded. Additionally, other applications for a more powerful laser do exist.

\section{E. Overall}

A complete design should be produced, including all necessary components-klystrons, cathode drive laser, 
refrigeration support, beam dump, etc. The commissioning process itself is likely to be divided into two main stagesthe electron beam and the x-ray beam. Initially, the electron beam will need to be produced at the intended interaction point, with the intended properties. Afterwards, the incident scattering laser will be installed, with the appropriate beam transport to allow the produced $\mathrm{x}$-ray beam to travel to the users and the electron beam to travel to the beam dump.

\section{SUMMARY}

The Compact ICLS design presented here would improve on all other compact sources to date, producing an X-ray beam of quality which is closer than ever to being comparable to beams produced at large-scale facilities. This is made possible by using $\mathrm{cw}$ superconducting $\mathrm{rf}$ to accelerate the beam before it is focused to the interaction point. At the interaction point, the electron beam has a small spot size and small transverse normalized rms emittance, which correspondingly result in an x-ray beam with high flux and brilliance. The ultralow emittance is made feasible by a low bunch charge, with a high repetition rate so the x-ray flux is not adversely affected.

This design achieves an electron bunch which generates an X-ray beam unmatched in quality by other Compact ICLS designs. These desired electron beam parameters are achieved by utilizing a number of different techniques. The most effective technique was the emittance compensation by $\mathrm{rf}$ focusing. By altering the geometry of the gun to provide the correct rf focusing for a given bunch, it is possible to produce bunches with low normalized transverse rms emittances. Taken together with the low bunch charge, the achieved transverse emittances are sufficiently small. Choosing the correct bunch length off the cathode is necessary, in order to produce a bunch exiting the linac which does not need compression, but is still long enough that the transverse space charge effects can be compensated for by the rf focusing provided by the gun geometry. Another beneficial technique is taking advantage of the quadrupole-like behavior of the double-spoke cavities which comprise the linac in order to produce a fairly round beam at the exit of the linac. An approximately round beam at the exit to the linac allows for the bunch to be easily focused down to a small spot size on the order of a few microns.

The use of superconducting cavities in this concept allows for a better electron beam at the interaction point, while keeping this concept compact. While the use of SRF cavities does increase the initial purchasing cost, it does reduce the long-term operating cost.

While the incident laser remains an incomplete component, its design should not be an obstacle. Further work outside of this aspect includes further optimization to improve on the current $\mathrm{x}$-ray parameters or altering the design for different functions-by increasing the x-ray energy, for example.

\section{ACKNOWLEDGMENTS}

The authors would like to thank Rocio Olave, Karim Hernández-Chahín, Subashini De Silva, Christopher Hopper, Randika Gamage, and Todd Satogata for earlier contributions to this project, and HyeKyoung Park for a discussion of cavity surface treatments. This material is based on work supported by the U.S. Department of Energy, Office of Science, Office of Nuclear Physics, Office of High Energy Physics, and Office of Basic Energy Science; and by the National Science Foundation. K. E. D and J. R. D. were supported at ODU by DOE Office of Nuclear Physics Award No. DE-SC00004094, DOE Office of High Energy Physics Award No. DE-SC0010081, and NSF Award No. 1416051. B. T. was supported at ODU by NSF Award No. 1535641. G. A. K was supported at Jefferson Lab by Contract No. DEAC05-06OR23177; additional support was provided by DOE Office of Nuclear Physics Award No. DESC004094 and Basic Energy Sciences Award No. JLABBES11-01. This research used resources of the National Energy Research Scientific Computing Center (NERSC), a U.S. Department of Energy Office of Science User Facility operated under Contract No. DE-AC02-05CH11231.

[1] W. C. Röntgen, On a new kind of rays, Nature (London) 53, 274 (1896).

[2] W. S. Graves, J. Bessuille, P. Brown, S. Carbajo, V. Dolgashev, K.-H. Hong, E. Ihloff, B. Khaykovich, H. Lin, K. Murari, E. A. Nanni, G. Resta, S. Tantawi, L. E. Zapata, F. X. Kärtner, and D. E. Moncton, Compact X-ray source based on burst-mode inverse Compton scattering at 100 kHz, Phys. Rev. ST Accel. Beams 17, 120701 (2014).

[3] M.-E. Couprie and J.-M. Filhol, X radiation sources based on acceleratorsSources de rayonnement $\mathrm{X}$ reposant sur l'emploi d'accélérateurs, C. R. Physique 9, 487 (2008).

[4] M. Jacquet, High intensity compact Compton X-ray sources: Challenges and potential of applications, Nucl. Instrum. Methods B 331, 1 (2014).

[5] G. A. Krafft and G. Priebe, Compton sources of electromagnetic radiation, Rev. Accel. Sci. Techol. 03, 147 (2010).

[6] K. Deitrick, J. R. Delayen, and G. A. Krafft, High average brilliance compact inverse Compton light source, in Proceedings of the 8th International Particle Accelerator Conference, IPAC 2017, Copenhagen, Denmark (JACoW, Geneva, Switzerland, 2017), p. 932, http://jacow.org/ ipac2017/papers/mopva036.pdf.

[7] J. Clarke, The Science and Technology of Undulators and Wigglers, Oxford Series on Synchrotron Radiation (Oxford University Press, New York, 2004).

[8] P. Duke, Synchrotron Radiation: Production and Properties (Oxford Science, New York, 2000).

[9] K. Deitrick, J. R. Delayen, B. R. P. Gamage, K. Hernandez, C. Hopper, G. A. Krafft, R. Olave, and T. Satogata, The ODU CAS inverse Compton source design, http:// toddsatogata.net/Papers/2013-09-03-ComptonSource-2up .pdf, (2013). 
[10] G. A. Krafft, E. Johnson, K. Deitrick, B. Terzić, R. Kelmar, T. Hodges, W. Melnitchouk, and J. R. Delayen, Laser pulsing in linear Compton scattering, Phys. Rev. Accel. Beams 19, 121302 (2016).

[11] K. Deitrick, Inverse Compton light source: A compact design proposal, Ph.D. thesis, Old Dominion University, 2017.

[12] N. Ranjan, B. Terzić, G. A. Krafft, V. Petrillo, I. Drebot, and L. Serafini, Simulation of inverse Compton scattering and its implications on the scattered linewidth, Phys. Rev. Accel. Beams 21, 030701 (2018).

[13] H. Wiedemann, Particle Accelerator Physics (Springer, New York, 2007).

[14] A. Chao, K. Mess, M. Tigner, and F. Zimmermann, Handbook of Accelerator Physics and Engineering (World Scientific, Singapore, 2013).

[15] A. Arnold and J. Teichert, Overview on superconducting photoinjectors, Phys. Rev. ST Accel. Beams 14, 024801 (2011).

[16] "Lyncean technologies, inc.-synchrotron light and the lyncean compact light source," http://www.lynceantech .com/technology.html.

[17] E. Eggl, M. Dierolf, K. Achterhold, C. Jud, B. Günther, E. Braig, B. Gleich, and F. Pfeiffer, The Munich Compact Light Source: initial performance measures, J. Synchrotron Radiat. 23, 1137 (2016).

[18] "Beamlines directory—advanced photon source," http:// www.aps.anl.gov/Beamlines/Directory.

[19] S. Benson, M. Borland, D. Douglas, D. Dowell, C. Hernandez-Garcia, D. Kayran, G. Krafft, R. Legg, E. Moog, T. Obina, R. Rimmer, and V. Yakimenko, X-ray sources by energy recovered linacs and their needed R\&D, Nucl. Instrum. Methods Phys. Res., Sect. A 637, 1 (2011).

[20] J. R. Delayen, Applications of spoke cavities, in Proceedings of the 2010 Linear Accelerator Conference, LINAC10, Tsukuba, Japan (JACoW, Geneva, Switzerland, 2010), p. 377, http://jacow.org/LINAC2010/papers/tu302.pdf.

[21] J. R. Delayen, Superconducting spoke cavities for electrons and high-velocity proton linacs, in Proceedings of the 2012 Linear Accelerator Conference, LINAC12, Tel Aviv, Israel (JACoW, Geneva, Switzerland, 2012), p. 758, http://jacow .org/LINAC2012/papers/th1a03.pdf.

[22] C. S. Hopper, H. Park, and J. R. Delayen, Cryogenic test of high-velocity spoke cavities, in Proceedings of the 2014 Linear Accelerator Conference, LINAC14, Geneva, Switzerland (JACoW, Geneva, Switzerland, 2014), p. 677, http:// jacow.org/LINAC2014/papers/tupp109.pdf.

[23] C. S. Hopper, H. Park, and J. R. Delayen, Development and testing of a $325 \mathrm{MHz} \beta_{0}=0.82$ single-spoke cavity, in Proceedings of the 2015 International Conference on RF Superconductivity, SRF 2015, Whistler, Canada (JACoW, Geneva, Switzerland, 2015), p. 744, http://srf2015.vrws .de/papers/tupb071.pdf.

[24] H. Park, C. S. Hopper, and J. R. Delayen, Fabrication and measurement of $500 \mathrm{MHz}$ superconducting double-spoke cavity, in Proceedings of the 2014 Linear Accelerator Conference, LINAC14, Geneva, Switzerland (JACoW, Geneva, Switzerland, 2014), p. 385, http://jacow.org/ LINAC2014/papers/mopp138.pdf.
[25] C. S. Hopper, K. Deitrick, and J. R. Delayen, Geometry effects on multipole components and beam optics in highvelocity multi-spoke cavities, in Proceedings of the 2013 North American Particle Accelerator Conference, NAPAC2013, Pasadena, CA, USA (JACoW, Geneva, Switzerland, 2013), p. 868, http://jacow.org/PAC2013/papers/ wepac42.pdf.

[26] W. Graves, W. Brown, F. Kaertner, and D. Moncton, MIT inverse Compton source concept, Nucl. Instrum. Methods Phys. Res., Sect. A 608, S103 (2009).

[27] M. A. Furman, Hourglass effects for asymmetric colliders, in Proceedings of Particle Accelerator Conference, PAC91, San Francisco, CA, USA, Particle Accelerator Conference No. 14 (IEEE, Piscataway, NJ, USA, 1991), p. 422, http://accelconf.web.cern.ch/AccelConf/p91/PDF/ PAC1991_0422.PDF.

[28] M. Jacquet and P. Suortti, Radiation therapy at compact Compton sources, Physica Medica 31, 596 (2015).

[29] P. Yu and W. Huang, Lattice design and beam dynamics in a compact X-ray source based on Compton scattering, Nucl. Instrum. Methods Phys. Res., Sect. A 592, 1 (2008).

[30] I. Drebot et al., Optimisation study of the Fabry-Pérot optical cavity For the MARIS/BriXS Compton x-ray source, in Proceedings of the 9th International Particle Accelerator Conference, IPAC 2018, Vancouver, BC, Canada (JACoW, Geneva, Switzerland, 2018), http:// ipac2018.vrws.de/papers/thpmf056.pdf.

[31] E. G. Bessonov, M. V. Gorbunkov, P. V. Kostryukov, Y. Y. Maslova, V. G. Tunkin, A. A. Postnov, A. A. Mikhailichenko, V. I. Shvedunov, B.S. Ishkhanov, and A. V. Vinogradov, Design study of compact Laser-Electron X-ray Generator for material and life sciences applications, J. Instrum. 4, P07017 (2009).

[32] A. Variola (ThomX Collaboration), The ThomX project, in Proceedings of the 2nd International Particle Accelerator Conference, IPAC'11, San Sebastian, Spain (JACoW, Geneva, Switzerland, 2011), p. 1903, http://jacow.org/ ipac2011/papers/weoaa01.pdf.

[33] A. Variola et al. (ThomX Collaboration), The ThomX project status, in Proceedings of International Particle Accelerator Conference (IPAC'14), Dresden, Germany, 2014, International Particle Accelerator Conference No. 5 (JACoW, Geneva, Switzerland, 2014), p. 2062, http:// jacow.org/IPAC2014/papers/wepro052.pdf.

[34] J. Urakawa, Development of a compact X-ray source based on Compton scattering using a $1.3 \mathrm{GHz}$ superconducting RF accelerating linac and a new laser storage cavity, Nucl. Instrum. Methods Phys. Res., Sect. A 637, S47 (2011).

[35] R. Hajima, N. Kikuzawa, N. Nishimori, T. Hayakawa, T. Shizuma, K. Kawase, M. Kando, E. Minehara, H. Toyokawa, and H. Ohgaki, Detection of radioactive isotopes by using laser Compton scattered $\gamma$-ray beams, Nucl. Instrum. Methods Phys. Res., Sect. A 608, S57 (2009).

[36] E. Bulyak, P. Gladkikh, A. Zelinsky, I. Karnaukhov, S. Kononenko, V. Lapshin, A. Mytsykov, Y. Telegin, A. Khodyachikh, A. Shcherbakov, V. Molodkin, V. Nemoshkalenko, and A. Shpak, Compact X-ray source based on Compton backscattering, Nucl. Instrum. Methods Phys. Res., Sect. A 487, 241 (2002). 
[37] B. R. P. Gamage and T. Satogata, Magnetic bunch compression for a compact Compton source, in Proceedings of the 2013 North American Particle Accelerator Conference, NAPAC2013, Pasadena, CA, USA (JACoW, Geneva, Switzerland, 2013), p. 1346, http://accelconf.web.cern .ch/AccelConf/PAC2013/papers/thpho21.pdf.

[38] A. Michalke, Photocathodes inside superconducting cavities-studies on the feasibility of a superconducting photoemission source of high brightness, Ph.D. thesis, Bergische Universität Gesamthochschule Wuppertal, 1993.

[39] A. Michalke, H. Piel, C. Sinclair, P. Michelato, C. Pagani, L. Serafini, and M. Peiniger, Photocathodes inside superconducting cavities: An experimetal approach to a superconducting photoemission source of high brightness, in Proceedings of the 5th Workshop on RF Superconductivity, SRF1991, Hamburg, Germany (JACoW, Geneva, Switzerland, 1991), p. 734, http://jacow.org/srf91/papers/srf91f16 .pdf.

[40] A. Michalke, H. Piel, C. Sinclair, and P. Michelato, First operation of high-quantum efficiency photocathodes inside superconducting cavities, in Proceedings of the 3rd European Particle Accelerator Conference, EPAC1992, Berlin, Germany (JACoW, Geneva, Switzerland, 1992), p. 1014, http://jacow.org/e92/PDF/EPAC1992_1014.PDF.

[41] C. Pagani, P. Michelato, and L. Serafini, High brightness, long pulse, electron beam production with SC photo-injectors, Nucl Instrum. Methods Phys. Res., Sect. A 340, 17 (1994).

[42] D. Janssen et al., First operation of a superconducting RF-gun, Nucl. Instrum. Methods Phys. Res., Sect. A 507, 314 (2003).

[43] J. R. Harris, K. L. Ferguson, J. W. Lewellen, S. P. Niles, B. Rusnak, R. L. Swent, W. B. Colson, T. I. Smith, C. H. Boulware, T. L. Grimm, P. R. Cunningham, M. S. Curtin, D. C. Miccolis, D. J. Sox, and W. S. Graves, Design and operation of a superconducting quarter-wave electron gun, Phys. Rev. ST Accel. Beams 14, 053501 (2011).

[44] F. Sannibale et al., Advanced photoinjector experiment photogun commissioning results, Phys. Rev. ST Accel. Beams 15, 103501 (2012).

[45] C. Gulliford, A. Bartnik, I. Bazarov, L. Cultrera, J. Dobbins, B. Dunham, F. Gonzalez, S. Karkare, H. Lee, H. Li, Y. Li, X. Liu, J. Maxson, C. Nguyen, K. Smolenski, and Z. Zhao, Demonstration of low emittance in the Cornell energy recovery linac injector prototype, Phys. Rev. ST Accel. Beams 16, 073401 (2013).

[46] B. Carlsten, New photoelectric injector design for the Los Alamos National Laboratory XUV FEL accelerator, Nucl. Instrum. Methods Phys. Res., Sect. A 285, 313 (1989).

[47] L. Serafini and J. B. Rosenzweig, Envelope analysis of intense relativistic quasilaminar beams in rf photoinjectors: mA theory of emittance compensation, Phys. Rev. E 55, 7565 (1997).

[48] V. Kumar, Understanding the focusing of charged particle beams in a solenoid magnetic field, Am. J. Phys. 77, 737 (2009).

[49] T. Wangler, RF Linear Accelerators (John Wiley and Sons, Inc., New York, 2008).

[50] D. Janssen and V. Volkov, RF focussing - an instrument for beam quality improvement in superconducting RF guns, Nucl. Instrum. Methods Phys. Res., Sect. A 452, 34 (2000).
[51] R. Akre, D. Dowell, P. Emma, J. Frisch, S. Gilevich, G. Hays, P. Hering, R. Iverson, C. Limborg-Deprey, H. Loos, A. Miahnahri, J. Schmerge, J. Turner, J. Welch, W. White, and J. Wu, Commissioning the Linac Coherent Light Source injector, Phys. Rev. ST Accel. Beams 11, 030703 (2008).

[52] D. H. Dowell, J. Castro, P. Emma, J. Frisch, S. Gilevich, G. Hays, P. Hering, C. Limborg-Deprey, H.Loos, A. Miahnahri, and W. White, LCLS injector drive laser, in Proceedings of Particle Accelerator Conference, PAC07, Albuquerque, NM, USA, Particle Accelerator Conference No. 22 (IEEE, Piscataway, NJ, USA, 2007), p. 1317, http://accelconf.web .cern.ch/AccelConf/p07/PAPERS/TUPMS058.PDF.

[53] Y. Park, M. Kulishov, R. Slavík, and J. Azaña, Picosecond and sub-picosecond flat-top pulse generation using uniform long-period fiber gratings, Opt. Express 14, 12670 (2006).

[54] S. Chaitanya Kumar and M. Ebrahim-Zadeh, Yb-fiberbased, high-average-power, high-repetition-rate, picosecond source at 2.1 $\mu \mathrm{m}$, Laser Photonics Rev. 10, 970 (2016).

[55] N. Apury Chaitanya, A. Aadhi, M. V. Jabir, and G. K. Samanta, High-power, high-repetition-rate, Yb-fiber laser based femtosecond source at $355 \mathrm{~nm}$, Opt. Lett. 40, 4269 (2015).

[56] S. Latkowski, R. Maldonado-Basilio, and P. Landais, Sub-picosecond pulse generation by $40-\mathrm{GHz}$ passively mode-locked quantum-dash 1-mm-long Fabry-Pérot laser diode, Opt. Express 17, 19166 (2009).

[57] M. G. Tarallo, J. Miller, J. Agresti, E. D’Ambrosio, R. DeSalvo, D. Forest, B. Lagrange, J. M. Mackowsky, C. Michel, J. L. Montorio, N. Morgado, L. Pinard, A. Remilleux, B. Simoni, and P. Willems, Generation of a flat-top laser beam for gravitational wave detectors by means of a nonspherical Fabry-Perot resonator, Appl. Opt. 46, 6648 (2007).

[58] J. Qiang, S. Lidia, R. D. Ryne, and C. Limborg-Deprey, Three-dimensional quasistatic model for high brightness beam dynamics simulation, Phys. Rev. ST Accel. Beams 9, 044204 (2006).

[59] "Poisson superfish," http://laacg.lanl.gov/laacg/services/ download_sf.phtml, 2012.

[60] C. S. Hopper, R. G. Olave, and J. R. Delayen, Superconducting single-spoke cavities for high-velocity applications, in Proceedings of the 4th International Particle Accelerator Conference, IPAC 2013, Shanghai,China (JACoW, Geneva, Switzerland, 2013), p. 2480, http:// jacow.org/IPAC2013/papers/wepwo079.pdf.

[61] C. S. Hopper, R. G. Olave, and J. R. Delayen, Development of spoke cavities for high-velocity applications, in Proceedings of the 3rd International Particle Accelerator Conference, IPAC 2012, New Orleans, LA, USA (JACoW, Geneva, Switzerland, 2012), p. 2456, http://jacow.org/ IPAC2012/papers/weppc103.pdf.

[62] C. S. Hopper and J. R. Delayen, Design of superconducting spoke cavities for high-velocity applications, in Proceedings of the 15th International Conference of RF Superconductivity, SRF2011, Chicago, IL, USA (JACoW, Geneva, Switzerland, 2011), p. 154, http://jacow.org/ SRF2011/papers/mopo033.pdf.

[63] J. R. Delayen, S. U. De Silva, and C. S. Hopper, Design of superconducting spoke cavities for high-velocity applications, in Proceedings of the 2011 Particle Accelerator 
Conference, PAC 2011, New York, NY, USA (JACoW, Geneva, Switzerland, 2011), p. 1024, http://jacow.org/ PAC2011/papers/tup100.pdf.

[64] C.S. Hopper and J. R. Delayen, Superconducting spoke cavities for high-velocity applications, Phys. Rev. ST Accel. Beams 16, 102001 (2013).

[65] C.S. Hopper, Development of superconducting spoke cavities for high-velocity applications, Ph.D. thesis, Old Dominion University, 2015.

[66] “Computer simulation technology website," http://www.cst .com.

[67] R. G. Olave, J. R. Delayen, and C. S. Hopper, Multipole expansion of the fields in superconducting high-velocity spoke cavities, in Proceedings of the 2012 Linear Accelerator Conference, LINAC12, Tel Aviv, Israel (JACoW, Geneva, Switzerland, 2012), p. 345, http://jacow.org/ LINAC2012/papers/mopb072.pdf.

[68] M. Borland, elegant: A Flexible SDDS-Compliant Code for Accelerator Simulation, Advanced Photon Source LS-287, September 2000.

[69] A. Grassellino, A. Romanenko, D. Sergatskov, O. Melnychuk, Y. Trenikhina, A. Crawford, A. Rowe, M. Wong, T. Khabiboulline, and F. Barkov, Nitrogen and argon doping of niobium for superconducting radio frequency cavities: a pathway to highly efficient accelerating structures, Supercond. Sci. Technol. 26, 102001 (2013).

[70] A. Grassellino, A. Romanenko, Y. Trenikhina, M. Checchin, M. Martinello, O. S. Melnychuk, S. Chandrasekaran, D. A. Sergatsov, S. Posen, A. C. Crawford, S. Aderhold, and D. Rice, Unprecedented quality factors at accelerating gradients up to $45 \mathrm{MVm}^{-1}$ in niobium superconducting resonators via low temperature nitrogen infusion, Supercond. Sci. Technol. 30, 094004 (2017).

[71] S. Posen, M. Liepe, and D. L. Hall, Proof-of-principle demonstration of $\mathrm{Nb}_{3} \mathrm{Sn}$ superconducting radiofrequency cavities for high Q0 applications, Appl. Phys. Lett. 106, 082601 (2015).

[72] R. Kephart, S. Biedron, B. Chase, S. Chattopadhyay, I. Gonin, A. Grassellino, S. Kazakov, T. Khabiboulline, S. Milton, S. Nagaitsev, R. Pasquinelli, P. Piot, S. Posen, O. Pronitchev, A. Romanenko, N. Sipahi, and V. Yakovlev, SRF, compact accelerators for industry \& society, in Proceedings of International Conference on RF Superconductivity, SRF2015, Whistler, BC, Canada (JACoW, Geneva, Switzerland, 2015), p. 1467, http://jacow.org/ srf2015/papers/frba03.pdf. 